\title{
Causal cortical dynamics of a predictive enhancement of speech intelligibility
}

DOI:

10.1016/j.neuroimage.2017.10.066

\section{Document Version}

Accepted author manuscript

Link to publication record in Manchester Research Explorer

\section{Citation for published version (APA):}

Di Liberto, G. M., Lalor, E. C., \& Millman, R. (2018). Causal cortical dynamics of a predictive enhancement of speech intelligibility. Neurolmage, 166, 247-258. https://doi.org/10.1016/j.neuroimage.2017.10.066

\section{Published in:}

Neurolmage

\section{Citing this paper}

Please note that where the full-text provided on Manchester Research Explorer is the Author Accepted Manuscript or Proof version this may differ from the final Published version. If citing, it is advised that you check and use the publisher's definitive version.

\section{General rights}

Copyright and moral rights for the publications made accessible in the Research Explorer are retained by the authors and/or other copyright owners and it is a condition of accessing publications that users recognise and abide by the legal requirements associated with these rights.

\section{Takedown policy}

If you believe that this document breaches copyright please refer to the University of Manchester's Takedown Procedures [http://man.ac.uk/04Y6Bo] or contact uml.scholarlycommunications@manchester.ac.uk providing relevant details, so we can investigate your claim.

\section{OPEN ACCESS}


Elsevier Editorial System(tm) for NeuroImage Manuscript Draft

Manuscript Number: NIMG-17-876R2

Title: Causal cortical dynamics of a predictive enhancement of speech intelligibility

Article Type: Full length article

Section/Category: Communication/learning/Language

Corresponding Author: Mr. Giovanni Di Liberto,

Corresponding Author's Institution: University of Dublin, Trinity College

First Author: Giovanni Di Liberto

Order of Authors: Giovanni Di Liberto; Edmund C Lalor, PhD; Rebecca E Millman, PhD

Abstract: Speech perception may be underpinned by a hierarchical cortical system, which attempts to match "external" incoming sensory inputs with "internal" top-down predictions. Prior knowledge modulates internal predictions of an upcoming stimulus and exerts its effects in temporal and inferior frontal cortex. Here, we used source-space magnetoencephalography (MEG) to study the spatiotemporal dynamics underpinning the integration of prior knowledge in the speech processing network. Prior knowledge was manipulated to i) increase the perceived intelligibility of speech sentences, and ii) dissociate the perceptual effects of changes in speech intelligibility from acoustical differences in speech stimuli. Cortical entrainment to the speech temporal envelope, which accounts for neural activity specifically related to sensory information, was affected by prior knowledge: This effect emerged early ( $50 \mathrm{~ms}$ ) in left inferior frontal gyrus (IFG) and then ( 100 ms) in Heschl's gyrus (HG), and was sustained until latencies of $250 \mathrm{~ms}$. Directed transfer function (DTF) measures were used for estimating direct Granger causal relations between locations of interest. In line with the cortical entrainment result, this analysis indicated that prior knowledge enhanced top-down connections from left IFG to all the left temporal areas of interest - namely HG, superior temporal sulcus (STS), and middle temporal gyrus (MTG). In addition, intelligible speech increased top-down information flow between left STS and left HG, and increases bottom-up flow in higher-order temporal cortex, specifically between STS and MTG. Together these results provide a detailed view of how, where and when prior knowledge influences continuous speech perception and they are compatible with theories that explain this mechanism as a result of both ascending and descending cortical interactions, such as predictive coding. 


\section{UNIVERSITY OF DUBLIN \\ TRINITY COLLEGE}

School of Engineering, Trinity Centre for Bioengineering and

Trinity College Institute of Neuroscience,

Trinity College Dublin,

Dublin 2, Ireland

Telephone: +353-87-7112972

Email: $\quad$ diliberg@tcd.ie

October $4^{\text {th }}, 2017$

Ref: Ms. No. NIMG-17-876R2

Dear Editors,

Would you please consider our revised manuscript for publication in NeuroImage? Our manuscript is entitled "Causal cortical dynamics of a predictive enhancement of speech intelligibility".

In our revised paper we have sought to address the concerns of the reviewers as diligently as possible. We are grateful to the reviewers for their efforts in critiquing our work, which we believe have helped us to greatly strengthen our manuscript.

Some of the main points in the revised version include:

1. The 'interaction contrast' cortical entrainment result (previously 'normalized difference') is now presented as a main figure, while the result for the individual conditions is now a supplementary information;

2. an improved statistical analysis and a more in-depth explanation of the statistical methods;

3. a more complete explanation of the weaker results for the connectivity analysis;

4. additional discussion points, including comments on the drawbacks of the specific experimental paradigm.

We have included a detailed point-by-point reply to the reviewers with our resubmission. Also, we highlighted in red the text that was modified.

Thank you for your consideration of our work.

Giovanni M. Di Liberto, Edmund C. Lalor \& Rebecca E. Millman. 


\section{$\underline{\text { Scientific grounds for consideration: }}$}

It is widely acknowledged that speech recognition is underpinned by a hierarchical cortical system. This system may be characterised by constant attempts to match incoming sensory input with top-down predictions. In real-world environments this active processing is particularly important as successful speech comprehension must contend with the enormous spectro-temporal variability of conversational speech and the various types of noise and speech degradation that characterize such environments. In these contexts, speech perception depends on both "external" sensory inputs and "internal" predictive mechanisms (Sohoglu et al., J Neurosci, 2012). A number of recent studies have measured increases and decreases in cortical activity in temporal and inferior frontal cortex while modulating these internal predictions by providing prior knowledge of an upcoming stimulus (Tuennerhoff \& Noppeney, NeuroImage, 2016). Although these effects have been discussed in the context of the possible integration between prior information and sensory input, the precise dynamics that underpin this phenomenon remain unclear.

Here, we use source-space magnetoencephalography (MEG) to study the spatiotemporal dynamics underpinning the integration of prior knowledge in the speech comprehension network. In particular, we examine how the online encoding of the speech envelope is affected when the intelligibility of degraded speech is enhanced by the provision of prior knowledge. Our results indicate that cortical entrainment to the speech temporal envelope, which accounts for neural activity specifically related to sensory information, is affected by prior knowledge: This effect emerged early in left inferior frontal gyrus, and then bottom-up propagated from primary auditory cortex to superior and middle temporal areas within the left hemisphere. A connectivity analyses demonstrated that intelligible speech increases top-down causality between low-order temporal areas and increases bottom-up causality in higher-order temporal cortex. Together these results are in line with previous work (Blank \& Davis, PLoS Bio., 2016), but go beyond that work by showing how, where and when prior knowledge influences speech perception. We contend that this work will be of interest not only to speech researchers, but to a broader audience interested in how top-down information interacts with bottom-up sensory input during perception. As such, we feel that NeuroImage would be an excellent venue for publicising our findings.

Thank you for your consideration of our work.

Giovanni M. Di Liberto, Edmund C. Lalor \& Rebecca E. Millman. 


\section{$\underline{\text { Abstract }}$}

Speech perception may be underpinned by a hierarchical cortical system, which attempts to match "external" incoming sensory inputs with "internal" top-down predictions. Prior knowledge modulates internal predictions of an upcoming stimulus and exerts its effects in temporal and inferior frontal cortex. Here, we used source-space magnetoencephalography (MEG) to study the spatiotemporal dynamics underpinning the integration of prior knowledge in the speech processing network. Prior knowledge was manipulated to i) increase the perceived intelligibility of speech sentences, and ii) dissociate the perceptual effects of changes in speech intelligibility from acoustical differences in speech stimuli. Cortical entrainment to the speech temporal envelope, which accounts for neural activity specifically related to sensory information, was affected by prior knowledge: This effect emerged early ( $\sim 50 \mathrm{~ms})$ in left inferior frontal gyrus (IFG) and then ( 100 ms) in Heschl's gyrus (HG), and was sustained until latencies of $\sim 250 \mathrm{~ms}$. Directed transfer function (DTF) measures were used for estimating direct Granger causal relations between locations of interest. In line with the cortical entrainment result, this analysis indicated that prior knowledge enhanced top-down connections from left IFG to all the left temporal areas of interest - namely HG, superior temporal sulcus (STS), and middle temporal gyrus (MTG). In addition, intelligible speech increased top-down information flow between left STS and left HG, and increases bottom-up flow in higher-order temporal cortex, specifically between STS and MTG. Together these results provide a detailed view of how, where and when prior knowledge influences continuous speech perception and they are compatible with theories that explain this mechanism as a result of both ascending and descending cortical interactions, such as predictive coding. 


\section{Highlights}

- Cortical entrainment to the speech envelope is modulated by prior knowledge

- Prior knowledge enhances delta-band entrainment

- Envelope tracking in left IFG precedes the same effect in HG

- Intelligible speech modulates causal cortico-cortical dynamics between temporal areas 
We thank the editor and both reviewers for their insightful and helpful comments. We have endeavored to respond to them as fully as possible and believe that the manuscript is improved as a result. The main changes in the revised version of the manuscript have been highlighted in red font. Please note that the figures have been re-ordered as it was suggested by both reviewers. We address individual comments on a point-by-point basis below.

\section{Reviewer \#1: Revision}

I thank the authors for their efforts. The manuscript has improved in clarity, and will, in my opinion, ultimately be suitable for publication in NeuroImage. Nevertheless, I have a few concerns that the authors may want to address.

We thank the reviewer for this new set of comments. We have done our best to address the various points and we believe that the manuscript is improved as a result. The main changes in the revised version of the manuscript have been highlighted in red font. Also, Figures 4, S3, and S4 have been re-ordered. We address individual comments on a point-by-point basis below.

(1)

Training and Figure 1: The authors improved the methods section. However, descriptions in the text regarding the training could still be extended and match what is shown in Figure 1. Figure 1 seems to indicate that training was also done on tone-vocoded sentences. This was not mentioned in the methods description. I recommend, the authors make figure and methods more consistent. Could the authors also more clearly indicate whether training consisted of passive listening?

We agree with the reviewer that this section could match Figure 1 better. We have reworded parts of the 'Experimental paradigm' paragraph, which is now more precise and is a better description of the figure. The only difference that remains between the text and the figure is that the latter doesn't show the 'silent' and the 'intelligible' conditions. This allows the simplification of an otherwise overcomplicated Figure 1, that focuses now on the conditions of interest for the analyses that follow.

(2)

Line 283-289: The authors mention that they believe the reconstruction of speech envelopes based on MEG data should not be different for different speech stimuli. I believe this conclusion could be problematic, given that only one single stimulus was used for the unintelligible condition and only one single stimulus was used for the pop-out condition. The authors also write (line 195-196) that different field patterns are expected for different conditions. More critically, when using the normalized difference which controls for potential acoustic differences (and which the authors use for the other analyses) some of their reported effects change. I recommend providing Figure S4 in the main manuscript as this reflects the analysis that controls for potential acoustic differences between sentences. This is particularly important here, because the study utilizes only two sentences.

About lines 195-196, we agree that the text was not clear. We have reworded that sentence, which now clarifies that we conducted the beamforming procedure separately for different conditions to avoid bias at this analysis step, by avoiding making any assumptions on the similarity between different experimental conditions.

About the main part of the comment: We believe that the reconstruction accuracies for the two stimuli should not change within block 1 because, despite the use of two stimuli only, the speech sentences had very similar properties (same speaker, duration, amplitude and phonemic rate). However, we understand the reviewer's concern and we agree to show the result for the normalised difference as a main figure (previously Figure S4). Indeed, this does not change the main finding of this analysis (early enhancement of cortical tracking in IFG and afterwards in HG). We find this reorganisation helpful because it highlights this main finding first, while the bottom-up flow meas- 
ured only for block 2 is presented in a following paragraph as an additional finding. Although we still believe that the subtraction of block2-block1 introduces additional noise that is masking the otherwise significant effects in STS and MTG, we think that this change clarifies and highlights the main result.

An additional consideration on this point is that the sensitivity to effects in STS and MTG may be weaker than in HG also because such cortical sites have been linked to higher-level properties of speech than the acoustic envelope, e.g., phonological features (Mesgarani et al., Science, 2014; Di Liberto et al., Curr Bio, 2015; Blank \& Davis, PLOS Biology, 2016).

We thank the reviewer for this comment that we believe improved the presentation of this main result.

(3)

Line 466ff: I was wondering whether the authors could briefly mention that positive values mean an enhancement from pre (block 1) to post (block 2) and that negative values mean a suppressive effect. This would help the reader to understand Figure 5. The authors focus in their description of the results on the pop-out conditions only. However, it is striking that most times where the pop-out conditions led to a significant effect, the unintelligible condition showed the opposite pattern. The authors may want to expand a little here. Furthermore, the top-down effects from left IFG to other regions are hardly noticeable and relatively small compared to some other effects the authors do not discuss. For example: rightHG to leftIFG, rightHG to rightMTG, rightMTG to rightIFG, etc. I recommend the authors find a more balanced description in this section.

We thank the reviewer for this comment. We appreciate that the text focuses only on the effects that more directly link to our initial hypotheses, while other significant effects have been neglected. We expanded this section that presents now a more comprehensive description of this figure.

It is difficult to comment on the occurrence of opposite patterns for the two conditions (pop-out and unint). One reason can be found in the multifaceted nature of these measures, which reflect effects such as stimulus repetition and perceptual enhancement within a single value. For this reason, statistical analyses were conducted on the 'interaction contrast' measure (formerly called 'normalized difference'), which isolates the effects of prior knowledge related to the perceptual enhancement from others like the stimulus repetition. That contrast is the measure that we describe in that paragraph, while it is more difficult to interpret effects for the pop-out and unintelligible conditions separately, as they may reflect multiple effects.

Minor:

- $\quad$ In the introduction (line 96ff), reference to the Millman et al. 2015 study does not provide the results of the study, but only what kind of approaches were chosen. A brief sentence about the results might better motivate why an entrainment analysis was conducted here, although Millman et al. already investigated entrainment.

Thanks for this comment. We added a few words on the results of Millman et al. 2015.

- Line 195: I recommend the authors indicate whether they mean all 4 conditions or whether they collapsed across block 1 and block 2. Furthermore, it was not entirely clear why the authors would think that each condition would elicit a different field pattern. Maybe this could be extended. If so, could the region of interest approach then selectively miss relevant activity for some conditions (spatially located differently)? Furthermore, if each condition elicits a different field pattern, wouldn't that make it all the more necessary to calculate the normalized difference for all analyses? Maybe the authors meant something else here. I recommend revisiting the text in this paragraph. 
As previously mentioned in the reply to comment 2, we have re-phrased the sentence that is now clearer about the procedure and the motivations for running the beamforming procedure on each individual condition.

- $\quad$ Line 235: The authors likely meant seconds.

Well spotted, thanks for pointing this out!

- $\quad$ Figure 4 and S4: It seem that the authors removed outliers from their box-plots? There was no mention of this in the methods or figure caption. Did the authors not use those data points?

Good point. The outliers were shown in the plot to allow for a clearer visualisation of the result distribution. Statistical analyses were conducted on all data points (including the outliers). We added this information in the figure captions. Thanks for pointing out this oversight.

- $\quad$ Line 412 and 425: The authors provide huge degrees of freedom for their ANOVAs which suggests they used a fixed-effects analysis here. A fixed-effects analysis would be rather unconventional for these types of data. Shouldn't the factor block have N-1 degrees of freedom (i.e., 15 instead of 1280)?

We thank the reviewer for this comment.

We understand the reviewer's concern and we also run a repeated measures three-way ANOVA that may be more conventional in this type of analysis. This produced results with nSub-1 (15) degrees of freedom as mentioned by the reviewer. This analysis shows once again that there is a significant main effect of Block in delta-band. This instead is not true anymore for theta-band:

Delta-band, repeated measures three-way ANOVA:

$F(1,15)=7.45 ; p=0.016 ;$ Partial Eta Squared $=0.332$

Theta-band, repeated measures three-way ANOVA:

$F(1,15)=2.17 ; p=0.162 ;$ Partial Eta Squared $=0.126$.

The revised version of the manuscript reports the results of this repeated measures ANOVA. Thank you for this comment.

- $\quad$ Line 472: MEG -> probably MTG

That's right, thanks.

Reviewer \#2: The authors were very responsive to my comments however I do have further comments arising for the revised version of the manuscript.

Re: response to comment 1 .

The authors state that previous studies provide no insight into which oscillatory bands are implicated in Granger causal connections but there is theoretical and limited experimental work from the predictive coding literature (e.g. Sedley et al. 2016 elife; Arnal et al. Nat Neuro 2011; Bastos et al. 2012 Neuron). This literature makes experimental predictions about which oscillatory bands AND which inter-regional connections might be involved in prior knowledge effects on perception. I would not suggest that this is mentioned in the Introduction but it could be mentioned in the Discussion section since it would seem to me to be highly relevant to the current work.

We thank the reviewer for pointing out references that are highly relevant to the present study. We have added some considerations in the discussion session that we hope will help the reader to link our work to other findings in the context of the effects of prior knowledge on perception.

Re: response to comment 2. 
I would like to see some discussion of this in the paper- what the authors see as the pros and cons of using massed repetition and also how this aspect of their study might explain differences between the current effects and those reported in previous studies.

We have added some discussion of the pros and cons of using repeated presentation of the same sentence to generate the "pop-out" effect. We also provide a more detailed response below.

Some previous work used "pop-out" to measure the effects of perceptual learning and/or prior knowledge on the perception of single words (Sohoglu et al., 2012, 2016; Blank et al., 2016). The use of single word speech stimuli helps to minimise the duration of an experiment employing a popout paradigm but does not inform on the effects of prior knowledge on speech perception at the sentence level. One of the limitations of the experimental design used by Millman et al. (2015) was the repeated presentations of the speech sentences used for the conditions of interest i.e. Pop-out and Unintelligible. This aspect of Millman et al.'s design was necessary to measure the effects of popout on sentence-level speech perception while also keeping the duration of the MEG data acquisition within a reasonable limit (40 mins). Baltzell et al. (2017) noted that the design used by Millman et al. (2015) could have resulted in overlearning of these limited speech materials and were able to use the pop-out paradigm for sentence-level speech perception where unique sentences were presented on each trial in an EEG experiment. However, EEG offers some advantage over MEG for experiments that require long durations of data acquisition. For example, EEG sensors move with the participant's head and therefore participants can move more freely during EEG recordings, which allows for longer experiments.

Another interesting observations is that this paradigm did produce results mainly in delta- and, marginally, theta-bands, while prior studies in the literature measured strong effects also in, for example, beta- and gamma-bands. The limited sensitivity to effects for high frequencies may be related to our experimental choice: While Arnal et al. (2011) used "valid" and "invalid" priors and showed an increase of oscillations in those bands for invalid prior, the present study used repeated stimuli with "valid" and "no" priors (pop-out and unintelligible conditions respectively).

Re: response to comment 3.

My understanding is that the new ANOVA was conducted on the interaction contrast ([Popout_post-Unintelligble_post]- ([Pop-out_pre-Unintelligble_pre])? Could the authors confirm? If so, I think for clarity this can be made even more explicit as to what dependent measure the ANOVA was conducted on. In the current version of the manuscript, at the point of describing the ANOVA, the dependent measure isn't currently referred to so in my opinion this could be made clearer.

I am still uneasy about presenting the separate Pop-out_pre-Unintelligble_pre and Pop-out_postUnintelligble_post contrasts in the main Figure 4. I still do not think it is justified to draw any conclusions from differences in these separate contrasts unless the interaction contrast is significant first. I suggest Figure S4 is used as the main Figure 4. The separate Pop-out_pre-Unintelligble_pre and Pop-out_post-Unintelligble_post contrasts can then be presented as follow-up tests in a supplementary figure.

I also find the authors' terminology a little confusing. Why 'normalized difference' measure? Technically the measure is a 'difference of differences'. Of course this is a rather inelegant term so better to say 'interaction contrast'.

We clarified the information on the contrast measure used for the ANOVA analysis. Thanks for pointing this out.

Secondly, we understand the reviewer's concern and we now report the normalized difference (previously Figure S4) as the main result. Of course, we adapted the manuscript to match this reorganisation of the figures.

Finally, the term 'normalized difference' refers to the concept that the contrast of interest is the difference within block 2, while the contrast within block 1 is the baseline. We understand that this term may be confusing as we are not normalising the data in the mathematical sense, but rather cen- 
tering it to a baseline. We were happy to change the contrast name to 'interaction contrast', which sounds more appropriate. We thank the reviewer for this comment.

Other comments:

Line 361. For clarity, specify that the effect in this sentence involves a suppressive effect of prior knowledge. I suggest revising to "A different pattern of results was measured in IFG, which showed early ( 100-550 ms) left-lateralized effects suppression of cumulative power in <gamma>-band" Permutation tests. Please specify how clusters were defined. With the MaxSum method (default in FieldTrip)? What was the cluster forming threshold? And were the tests one-tailed or two-tailed?

We clarified the first point in the manuscript.

We have also added further details on the permutations tests in the 'Statistical analysis' section, thanks for pointing this out. Here, we adopted bespoke cluster-size statistics that identified the minimum cluster-size thresholds to consider a cluster significant (using Wilcoxon signed-rank tests; the cluster forming threshold was set to 0.05 ; furthermore, the result did not change when using a threshold of 0.01). The result was identical when running a cluster-mass statistics (MaxSum method that looks at the sum of t-values in each cluster), so we just reported one of the two tests (clustersize statistics). Finally, all tests were two-tailed. 
Causal cortical dynamics of a predictive enhancement of speech intelligibility

\author{
Giovanni M. Di Liberto ${ }^{1}$, Edmund C. Lalor ${ }^{1,2}$, Rebecca E. Millman ${ }^{3}$
}

$6{ }^{1}$ School of Engineering, Trinity Centre for Bioengineering, and Trinity College Institute of Neuroscience, Trinity College Dublin, Dublin, Ireland ${ }^{2}$ Department of Biomedical Engineering, Department of Neuroscience, and Del Monte Institute for Neuroscience, University of Rochester, Rochester

New York, 14627.
${ }^{3}$ Manchester Centre for Audiology and Deafness, Division of Human Communication, Development and Hearing, School of Health Sciences, Faculty of Biology, Medicine and Health, University of Manchester, UK.

Contact author:

G.D.L., 152-160 Pearse Street, Dublin 2, Ireland, +353-1-8961743, diliberg@tcd.ie

R.E.M., B2.8 Ellen Wilkinson Building, Oxford Road, Manchester, M13 9PL, UK, +44 161275 3387, rebecca.millman@manchester.ac.uk

Keywords: entrainment, speech intelligibility, magnetoencephalography, beamforming

Highlights:

- $\quad$ Cortical entrainment to the speech envelope is modulated by prior knowledge

- $\quad$ Prior knowledge enhances delta-band entrainment

- $\quad$ Enhanced envelope tracking in left IFG precedes the same effect in left HG

- Intelligible speech modulates causal cortico-cortical dynamics between temporal areas 


\section{Abstract}

Speech perception may be underpinned by a hierarchical cortical system, which attempts to match "external" incoming sensory inputs with "internal" top-down predictions. Prior knowledge modulates internal predictions of an upcoming stimulus and exerts its effects in temporal and inferior frontal cortex. Here, we used source-space magnetoencephalography (MEG) to study the spatiotemporal dynamics underpinning the integration of prior knowledge in the speech processing network. Prior knowledge was manipulated to i) increase the perceived intelligibility of speech sentences, and ii) dissociate the perceptual effects of changes in speech intelligibility from acoustical differences in speech stimuli. Cortical entrainment to the speech temporal envelope, which accounts for neural activity specifically related to sensory information, was affected by prior knowledge: This effect emerged early $(\sim 50 \mathrm{~ms})$ in left inferior frontal gyrus (IFG) and then $(\sim 100$ $\mathrm{ms}$ ) in Heschl's gyrus (HG), and was sustained until latencies of $250 \mathrm{~ms}$. Directed transfer function (DTF) measures were used for estimating direct Granger causal relations between locations of interest. In line with the cortical entrainment result, this analysis indicated that prior knowledge enhanced top-down connections from left IFG to all the left temporal areas of interest - namely HG, superior temporal sulcus (STS), and middle temporal gyrus (MTG). In addition, intelligible speech increased top-down information flow between left STS and left HG, and increases bottom-up flow in higher-order temporal cortex, specifically between STS and MTG. Together these results provide a detailed view of how, where and when prior knowledge influences continuous speech perception and they are compatible with theories that explain this mechanism as a result of both ascending and descending cortical interactions, such as predictive coding. 


\section{Introduction}

Humans have the ability to understand speech despite the various sources of noise and degradation that characterise real-world listening environments. Under perceptually adverse listening conditions, the perception of sensory information is aided by additional factors, such as the prior knowledge of the content of the upcoming speech (Obleser, 2014). A major challenge is to understand exactly how, where, and when those predictions influence speech perception (Norris et al., 2016). It is widely accepted that speech comprehension is underpinned by a hierarchical network that is characterized by both bottom-up and top-down signals (Hickok and Poeppel, 2007; Peelle et al., 2010; Gross et al., 2013; Bornkessel-Schlesewsky et al., 2015). In particular, top-down connections may constitute a neural basis for the integration of prior knowledge in the speech processing network (Davis and Johnsrude, 2007; Wild et al., 2012; Lewis and Bastiaansen, 2015).

The ability to disentangle neural activity at distinct processing levels may be crucial to unveil how prior information affects the speech comprehension network. One way to achieve this goal is to focus on the cortical organization of speech processing using neuroimaging technologies with high spatial resolution, such as functional magnetic resonance imaging (fMRI) (Friederici et al., 2010; DeWitt and Rauschecker, 2012; Overath et al., 2015; Tuennerhoff and Noppeney, 2016). Such studies have contributed to the characterization of specific cortical areas in terms of their functional roles in speech comprehension. In particular, a hierarchical organization of temporal areas supporting the perceptual and lexical processing of speech has been identified: Key regions include the superior temporal gyrus (STG) (Humphries et al., 2014) and the superior temporal sulcus (STS) (Overath et al., 2015), which exhibit sensitivity to acoustic and phonetic features of speech. Furthermore, the middle temporal gyrus (MTG) has been implicated in higher-level lexical processing (Lau et al., 2008; Turken and Dronkers, 2011).

The low temporal resolution of fMRI constitutes a major impediment when investigating the fast cortical dynamics of the speech processing network (Gow and Segawa, 2009; Wild et al., 2012). Complementary insights may be provided using electrocorticography (ECoG) and non-invasive electroencephalography (EEG) and magnetoencephalography (MEG), which are more suited to characterising the precise temporal dynamics required to integrate bottom-up and top-down information. In particular, ECoG studies have identified a role for STG in categorical perception and, specifically, in the processing of phonetic-level features (Chang et al., 2010; Mesgarani et al., 2014). ECoG, EEG and MEG have all been used to show that prior knowledge elicits a top-down influence from inferior frontal gyrus (IFG) to STG (Sohoglu et al., 2012; Leonard et al., 2016; Sohoglu and Davis, 2016). Although these studies provide important insights into the mechanisms of key regions in the speech network, a number of fundamental questions remain unanswered, especially regarding the temporal dynamics and interactions between cortical areas. Previous studies either did not have the spatial resolution (Di Liberto et al., in review), the temporal resolution (Blank and Davis, 2016; Tuennerhoff and Noppeney, 2016) or the cortical coverage (Holdgraf et al., 2016) to characterize precise spatiotemporal dynamics between regions in the speech network. Studies with the requisite spatiotemporal resolution (Sohoglu et al., 2012; Sohoglu and Davis, 2016), focused on cortical (de)activation, rather than indexing the representational content that may underlie such responses, i.e., the neural encoding of speech features.

Here, we sought a better understanding of the spatiotemporal cortical dynamics that underpin the integration of prior knowledge within the speech comprehension network. Importantly, this study aims to investigate these dynamics both in terms of changes in activity in key cortical areas and in terms of the neural encoding of the temporal envelope of speech. To this end, data from a perceptual "pop-out" experiment (Millman et al., 2015) were re-analyzed to isolate the effects of prior knowledge on cortical mechanisms supporting speech intelligibility. In Millman et al. (2015), 
perceptual "pop-out" (e.g. Davis et al., 2005) was used to change the percept of physically identical tone-carrier vocoded speech sentences (in short, tone-vocoded sentences) from unintelligible to intelligible during MEG data acquisition. The pop-out effect was obtained by preceding the presentation of some of the vocoded sentences with the original, unprocessed version of the stimulus. The pop-out approach dissociates the effects of (top-down) prior knowledge from (bottom-up) changes in sensory information (Sohoglu et al., 2012; Millman et al., 2015; Blank and Davis, 2016; Holdgraf et al., 2016; Sohoglu and Davis, 2016; Di Liberto et al., in review).

100 In order to assess how prior knowledge affects speech processing within the speech comprehension 101 network, bespoke MEG beamformer-based analyses were used to estimate neural sources in 102 bilateral locations of interest (Millman et al., 2015), corresponding to Heschl's gyrus (HG), STS, 103 MTG, and IFG. These regions have been shown to provide distinct contributions to the speech 104 recognition process and to represent progressively higher levels of the speech perception hierarchy 105 (Davis and Johnsrude, 2003; Scott and Johnsrude, 2003; Hickok and Poeppel, 2007; Peelle et al., 106 2010; Peelle et al., 2013; Mesgarani et al., 2014; Overath et al., 2015; Leonard et al., 2016; Sohoglu 107 and Davis, 2016; Tuennerhoff and Noppeney, 2016). The neural encoding of speech was estimated using measures of cortical entrainment to the temporal envelope of speech sentences (Lalor et al., 2009b; Crosse et al., 2016b). The functional roles and interpretations of the cortical entrainment phenomenon are still debated (Ding and Simon, 2014b) and, crucially, previous research (including an analysis of the same MEG dataset used in the present study) failed to reveal any significant effect of intelligibility on entrainment measures (Millman et al., 2015). Here, we investigated this mechanism by combining more sophisticated measures of the cortical tracking of speech (Lalor et al., 2009a; Crosse et al., 2016b), incorporating additional spatial, spectral, and temporal detail. Therefore, the primary goals of this study were to determine whether entrainment to the speech envelope i) is affected by perceptual pop-out, ii) entails sensitivity to the integration of prior knowledge with sensory information, iii) reflects the consequent change in perceived intelligibility. Secondly, we aimed to investigate the top-down/bottom-up dynamics of the pop-out effect by using measures of cortical entrainment, event-related power, and effective connectivity. 


\section{Methods}

The present study is based on new analyses of a previously published MEG study on perceptual "pop-out" (Millman et al., 2015).

\section{Participants}

Sixteen right-handed native English speakers (10 males; mean age $=29.2$ years $+/-7.8$ years, age range $=20-48$ years) took part in this experiment.

\section{Stimuli and procedure}

\section{Speech stimuli}

Short-duration sentences spoken by an adult British English male (BKB/IHR corpus; e.g. Macleod and Summerfield, 1987; Foster et al., 1993) were used as the speech stimuli. The duration of each speech sentence was approximately $1.5-\mathrm{s}$, and the duration of each epoch was extended to $2.5-\mathrm{s}$ through the addition of approximately 1 -s of silence to the end of each sentence. Stimuli were delivered diotically to participants via Etymotic insert earphones (Etymotic Research ER30, Elk Grove Village, IL) at a comfortable sound level.

The sentences were "The kettle boiled quickly" (always unintelligible), and "The floor was quite slippery" (the pop-out sentence), and "She ironed her skirt" (always intelligible). The intelligibility of all three speech sentences was degraded by using a tone-carrier vocoder (e.g. Dudley, 1939). A vocoder with only three carriers was used to produce tone-vocoded stimuli that were unintelligible prior to exposure to the original, unprocessed version of the same sentence. The carrier frequencies were 225,1047 , and $4861 \mathrm{~Hz}$. The temporal envelopes at the output of each channel were extracted using half-wave rectification and smoothing. The cut-off frequency of the low-pass filter used to smooth the extracted temporal envelope varied depending on the carrier frequency. Specifically, the cut-off frequency was set to half the equivalent rectangular bandwidth (e.g. Moore and Glasberg, 1983) of each channel (24, 68, and $274 \mathrm{~Hz}$ for each of the carrier frequencies, respectively). The temporal envelopes extracted from each band were then averaged to form the broadband speech temporal envelope for each sentence.

\section{Experimental paradigm}

The experiment was carried out in a single session for each subject and was composed of three parts. The main rationale was to present participants with unintelligible vocoded speech, which was perceived as unintelligible regardless of how many times it was repeated. Crucially, prior exposure to the original clear version of vocoded speech enhances perceived intelligibility when listening to the vocoded version (i.e., the "pop out" effect; Davis et al., 2005). In this experiment, participants were presented with paired repetitions of the original version of the Intelligible stimulus and its tone-vocoded counterpart at the beginning of the MEG session. During block 1, MEG data were recorded as participants listened to the three vocoded speech sentences and to silent trials (2.5-s duration). These auditory conditions were named Pop-out, Unintelligible, Intelligible, and Silent, and they were presented in random order for a total of 100, 100, 50, and 50 times respectively. In block 1, participants could comprehend only the Intelligible stimulus. At the end of block 1, participants were presented with repetitions of both the original and the tone-vocoded versions of the Pop-out stimulus (passive listening) during a training block with no neural recordings. Finally, 
MEG recordings were performed during block 2, which was physically the same as block 1, but with the crucial difference that both the Intelligible and the Pop-out stimuli were perceived as intelligible. Figure 1 shows the conditions of interest, i.e., Unintelligible and Pop-out, which are denoted as Pop-out pre and Unintelligible pre when they were recorded before the training block, and are referred to as Pop-out post $_{\text {and }}$ anintelligible ${ }_{\text {post }}$ when recorded after the training block. In addition, probe trials were presented during each block ( 2.5 seconds duration; 25 per block). During a probe trial, participants were played an auditory cue, which prompted them to respond, using a button box, and indicate a binary intelligibility rating (intelligible or unintelligible) for the last sound they heard. The resulting values were used to verify that the desired enhancement of perceived clarity from block 1 to block 2 occurred for the Pop-out trials but not for the Unintelligible trials. As reported in Millman et al. (2015), due to a technical issue, intelligibility ratings were only recorded for 15 of the 16 participants.

\section{MEG recordings}

Data were collected at the University of York, UK, using a Magnes 3600 whole-head 248-channel magnetometer (formerly 4-D Neuroimaging, Inc., San Diego, CA). The signals were recorded at a sample rate of $678.17 \mathrm{~Hz}$ and were low-pass filtered online with a cutoff frequency of $200 \mathrm{~Hz}$. Before recording, individual facial and scalp landmarks (left and right preauricular points, $\mathrm{Cz}$, nasion, and inion) were spatially coregistered using a Polhemus Fastrak System. The landmark locations in relation to the sensor positions were derived on the basis of a precise localization signal provided by five spatially distributed head coils with a fixed spatial relation to the landmarks. These head coils provided a measurement of a participant head movement at the beginning and end of each data acquisition block.

The raw data in each epoch were inspected visually. Epochs contaminated with either physiological or non-physiological artifacts were manually removed.

\section{Coregistration}

For the source-space analyses, the landmark locations were matched with the individual participants' anatomical magnetic resonance (MR) scans using a surface-matching technique adapted from Kozinska et al. (2001). T1-weighted MR images were acquired with a GE 3.0-T Signa Excite HDx system (General Electric, Milwaukee, WI) using an eight-channel head coil and a 3-D Fast Spoiled Gradient Recall sequence: repetition time/echo time/flip angle $=8.03 \mathrm{~ms} / 3.07 \mathrm{~ms} / 20^{\circ}$, spatial resolution of $1.13 \mathrm{~mm} \times 1.13 \mathrm{~mm} \times 1.0 \mathrm{~mm}$, in-plane resolution of $256 \times 256 \times 176$ contiguous slices. The individuals' data were spatially normalized to the Montreal Neurological Institute (MNI) standard brain, based on the average of 152 individual T1-weighted structural images (Evans et al., 1993). The source-space grid for each participant was initially defined in MNI space and linearly transformed back to individual MRIs.

\section{Beamformer-based analyses}

For further details on the beamformer-based analysis framework used in this study, please refer to Millman et al. (2015).

In brief, a vectorized, linearly constrained minimum-variance beamformer (Van Veen et al., 1997; Huang et al., 2004) was used to obtain the spatial filters with a multiple-spheres head model (Huang 
et al., 1999). Given that we expected different patterns of cortical activation in the two different experimental conditions of interest, this procedure was conducted separately for each condition. The beamformer grid size was $5 \mathrm{~mm}$. The three orthogonal spatial filters were implemented as a single 3-D system (see Johnson et al., 2011). In this beamformer framework, the orientation of each spatial filter is a crucial free parameter that should be specified for the metric of interest. In this study, an independent, unsupervised approach was chosen for the optimization of the spatial-filter orientation. In particular, a principal component analysis (PCA) was performed to extract one dominant signal from a space of 163 orientations with equal spatial distribution. This choice allowed for all analyses (power, envelope entrainment, and causality) to be performed using the same source-space data and avoided possible overfitting, due to the dependency between spatial-filter orientation and entrainment analysis parameters.

\section{Locations of interest}

The aim of this study was to characterize the effects of prior knowledge (and of the consequent enhancement in speech intelligibility) on the activity in, and interaction between, several bilateral key locations in the speech comprehension hierarchy (e.g. Hickok and Poeppel, 2007): These key locations included, as depicted in Figure 2, HG, STS, [MNI: $\pm 61,-22,0]$ (coordinates taken from Overath et al., 2015); posterior MTG, [MNI: $\pm 55,-46,-4]$ (coordinates taken from Lau et al., 2008); IFG, [MNI: $\pm 54,18,20$ ] (coordinates taken from Sohoglu et al., 2012). As in the work from Millman and colleagues (Millman et al., 2013; Millman et al., 2015), left and right HG were manually seeded because the anatomy of $\mathrm{HG}$ varies considerably among individuals (e.g. Rademacher et al., 2001).

\section{Frequency bands of interest}

Spatial filters from the LOIs were generated using a time window of $2000 \mathrm{~ms}$, including $500 \mathrm{~ms}$ prior to stimulus presentation. Broadband $(1-45 \mathrm{~Hz})$ data (obtained using $4^{\text {th }}$ order Butterworth filters) from the conditions of interest (Pop-out, Unintelligible) were projected through the spatial filters in the first instance so that all analyses (i.e., power envelope, entrainment and causality) could be carried out using the same spatial filter orientation. Contributions from more specific brain rhythms were assessed by spectrally filtering the broadband source-space signal in the frequency bands $\Delta$ (1-4 Hz), $\theta(4-8 \mathrm{~Hz}), \alpha(8-15 \mathrm{~Hz}), \beta(15-30 \mathrm{~Hz})$, and $\gamma(30-45 \mathrm{~Hz})$ using Chebyshev Type 2 digital filters in both a forwards and backwards direction to remove phase-distortion.

\section{Event-related power analyses}

Event-related fields time-locked to stimulus onset were derived for Pop-outpre, Pop-outpost, Unintelligiblepre, and Unintelligiblepost. Whilst fMRI studies are limited to overall measures of cortical activity over relatively long time windows, the current analysis also investigated the temporal dynamics of the cortical responses to speech. This information is conveyed by means of the cumulative event-related power, where cumulative power at time $t$ is calculated as the sum of the squares for the time window $[0, t]$. The use of this measure allowed for a clearer visualization of overall trends across the duration of a sentence compared to the more common point-by-point power analysis. Baseline correction was applied using the pre-stimulus time interval from -0.5 to $0.2 \mathrm{~s}$. 
measure was derived for each location and frequency band using the same contrast as for the 'interaction contrast' used by Millman et al. (2015): [(Pop-outpost-Pop-outpre $)$ - (Unintelligiblepost Unintelligiblepre)]. The reasoning behind the use of this specific contrast is that UnintelligiblepostUnintelligiblepre accounts for MEG differences due to repetition, while Pop-outpost - Pop-outpre also reveals the effects of prior knowledge and its effects on speech intelligibility. This overall measure reflects the changes in source-space MEG responses due to the perceptual pop-out. On a similar note, the same contrast could be re-written as [(Pop-outpost - Unintelligiblepost $)-($ Pop-outpre Unintelligiblepre)], where Pop-outpre - Unintelligiblepre accounts for MEG variation due to low-level physical differences between the two vocoded sentences, while Pop-outpost - Unintelligiblepost also reflects the effects of prior knowledge and its effects on speech intelligibility.

\section{Cortical entrainment analyses}

The mapping between stimulus and cortical activity was estimated using a system identification approach based on ridge-regression. In particular, the procedure involved identifying a mapping from source-space MEG signal to the speech envelope that optimized the following linear model:

$$
\hat{s}_{\text {loc }}(t)=\sum_{\tau=\tau_{i}}^{\tau_{i}+\text { winsize }} r(t+\tau, \text { loc }) g(\tau, \text { loc }),
$$

where $\hat{s}_{l o c}(t)$ is the estimated speech envelope using the MEG signal from a location of interest loc, $r(t+\tau, l o c)$ is the MEG response time lag $\tau_{i}$ and location loc, and $g(\tau, l o c)$ is the linear decoder for the corresponding time lag and location. The objective was to reconstruct the underlying speech envelope and to compare the quality of such reconstructions across experimental conditions and cortical locations of interest. The decoder $g(\tau, l o c)$ was optimized for each condition using leaveone-out cross-validation while maximizing the correlation between $\hat{s}_{l o c}(t)$ and $s(t)$ (Crosse et al., 2016b; mTRF Toolbox: http://sourceforge.net/projects/aespa/). At the cross-validation step $i$, data from all trials but trial ${ }_{i}$ were used to fit a model and to reconstruct an estimate of the envelope for $\operatorname{trial}_{i}$. The procedure was then repeated for all trials, which allowed us to derive such an estimate for every trial. Ridge-regression provides us with a regularization parameter $\lambda$ to prevent overfitting to the training data. Here, we conducted a parameter search to select the optimal $\lambda$ value, i.e., the $\lambda$ that produces the highest mean correlation between envelope and its estimates across all trials and all subjects. This mean correlation, which was measured by calculating Pearson's correlation $(r)$, represents a quantitative measure of cortical entrainment to the envelope of speech. Note that the optimization procedure was run separately for each condition and that the resulting optimal values of $\lambda$ were the same for each condition.

This approach, called the Temporal Response Function (TRF), allowed for the estimation of mappings from the MEG signal to the envelope of the stimulus (backward modeling approach) for each location and frequency band of interest. A window of time lags with a duration of $200 \mathrm{~ms}$ (Crosse et al., 2016b) was shifted from shorter to longer latencies with steps of $50 \mathrm{~ms}$ (from -50 $150 \mathrm{~ms}$ to $200-400 \mathrm{~ms}$ ), which allowed investigation of the spatiotemporal dynamics of the effect of interest. Note that models are fit on the whole duration of a sentence $(1.5 \mathrm{~s})$, while the time-lag window-size refers to how many data-points of the MEG signal are used to reconstruct one single point of the speech envelope. See (Puvvada and Simon, 2017) for a similar use of different timewindows in the context of selective attention to speech.

Here, the contrast used for the power analysis [(Pop-outpost - Unintelligiblepost $)-($ Pop-outpre Unintelligiblepre)] was decomposed into Pop-outpost - Unintelligiblepost and Pop-outpre Unintelligible pre. Only the Pop-outpost - Unintelligiblepost contrast involves the perceptual pop-out 
effect, while Pop-outpre - Unintelligiblepre may only reflect differences in cortical entrainment due to low-level physical differences between the two vocoded sentences. The choice of within-block contrast measures ensures that the resulting effects are neither due to repetition suppression nor perceptual learning. In fact such factors could emerge here only when the number of prior presentations of the stimuli differs between conditions, i.e., for contrasts between different blocks.

These within-block contrasts could not be used in the event-related power analysis, as the subtraction of MEG power would be dominated by low-level physical differences between the two sentences. This is not the case for the entrainment analysis, which is based on Pearson's correlations between speech envelopes and their reconstructions that were averaged across all trials for one given experimental condition. In fact, given input stimuli with similar spectro-temporal properties (e.g. same speaker and speech rate), the average ability to reconstruct their envelope was not expected to vary for different stimuli.

\section{Network effective connectivity analysis}

Brain connectivity measures are used to infer neuronal spatiotemporal interactions which index and predict task-relevant changes in cognitive states and behavior. Whilst methods such as dynamic causal modeling (DCM) require a set of possible hypotheses for the neurobiological system of interest (Stephan et al., 2007), there exist approaches that do not impose such a constraint and that rely on data-driven analyses (Granger, 1969; Ding et al., 2006). Here, an exploratory dynamical framework for neuronal system identification was used to assess the effect of prior information on the effective connectivity between cortical areas of interest in temporal and frontal lobes (effective connectivity denotes asymmetric causal dependences between brain regions). To this end, the source information flow toolbox (SIFT, Delorme et al., 2011) was used to investigate such causal effects in the context of bottom-up/top-down cortical information flow.

SIFT was used to fit Vieira-Morf models on the source-space MEG data. A direct directed transfer function (dDTF; Korzeniewska et al., 2003) measure was used to estimate the direct causality between pairs of cortical locations of interest ('directed' indicates that nodes are connected by directional edges; 'direct' clarifies that direct information flow is isolated from indirect/spurious edges). dDTF can be interpreted as a frequency-domain conditional Granger causality (GC) measure and is effective in removing spurious indirect causal influence between brain sources (Kus et al., 2004). In other words, one benefit of this approach is that it disentangles direct influence between nodes from spurious effects. For example, given a network with three interconnected nodes $\mathrm{A} \rightarrow \mathrm{B} \rightarrow \mathrm{C}$, a non-conditional approach could also report a spurious significant link $\mathrm{A} \rightarrow \mathrm{C}$, even though such a connection is indirect. For each connection, this analysis looks for significant causal interactions in the time-frequency domain. The SIFT toolbox was provided with the broad-band source-space data (1-45 Hz). Data preprocessing consisted of constant detrending, and time and ensemble normalization with the following model parameters: model order $=18$, window size $=300$ $\mathrm{ms}$, step size $=30 \mathrm{~ms}$. Furthermore, SIFT performs a frequency analysis by means of a segmentation-based linear vector autoregressive model (similar to a short-time Fourier transform) (Ding et al., 2000). Model validation was performed by checking for its stability and the whiteness of the residuals by means of the autocorrelation function (ACF) test (Lütkepohl, 2007; Delorme et al., 2011). The smallest model order that led to stability and whiteness for all experimental conditions was selected.

As for the event-related power analysis, the contrasts between (Pop-outpost - Pop-outpre) and (Unintelligible post - Unintelligiblepre) were used to investigate the effect of prior knowledge in the spatio-spectral MEG domain. Because the time domain was involved, the connectivity analysis was conducted first on the contrasts Pop-outpost - Pop-outpre and Unintelligiblepost - Unintelligiblepre 
333 (Figure S5), as they involved the same physical stimulus and, therefore, the time dimension could 334 be preserved. By collapsing the dDTF measure along the time domain, it was possible to compare 335 the results for Pop-out and Unintelligible stimuli (Figure 5). Paired Wilcoxon tests were used to 336 identify significant effects for the difference of the results for the two stimuli, which corresponds to 337 the normalized contrast measure.

\section{Statistical analysis}

339 All statistical analyses were conducted using Wilcoxon signed rank tests (paired if possible), except 340 where otherwise stated. All numerical values are reported as mean \pm SD. In the cortical power and 341 in the entrainment analyses, permutation-based cluster-size statistics (Groppe et al., 2011; Maris, 342 2012) with 1000 repetitions were used to correct for multiple comparisons while keeping in 343 consideration that results for neighboring time points or time windows are not independent. The 344 primary threshold of the cluster statistics was set to $p=0.05$ and all tests were two-tailed. Note that 345 identical results were obtained using cluster-mass statistics (Ing \& Schwarzbauer, 2014). In the 346 connectivity analysis, Bonferroni correction was applied by taking into consideration both the 347 number of frequency bins and the number of nodes. 


\section{Behavioral intelligibility ratings}

The responses made during the probe trials were analyzed to confirm that the Pop-out sentences were perceived as more intelligible in block 2, i.e., after exposure to the unprocessed speech. The low intelligibility ratings for the Pop-outpre $($ mean $=15.7 \%, \mathrm{SD}=34 \%)$, Unintelligiblepre $(\mathrm{mean}=$ $11.1 \%, \mathrm{SD}=17.3 \%)$, and Unintelligiblepost $($ mean $=17.8 \%, \mathrm{SD}=28.9 \%)$ sentences indicate that they were perceived as unintelligible. The intelligibility ratings for Pop-outpost (mean $=93.5 \%$, SD $=15.2 \%$ ) were significantly greater than the ones for Pop-outpre (Wilcoxon signed rank test, $N=15$

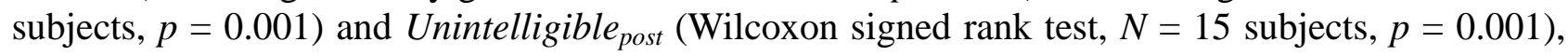
indicating that prior knowledge induced perceptual pop-out in the Pop-out condition only. In the Unintelligible control condition, the ratings for Unintelligible pre were not significantly different than those for Unintelligiblepost, (Wilcoxon signed rank test, $N=15$ subjects, $p=0.15$ ), indicating that the Unintelligible condition was an adequate control for temporal order effects. In block 1, there were no significant differences in the rating for Pop-out $t_{\text {pre }}$ and Unintelligible pre $_{\text {(Wilcoxon signed }}$ rank test, $N=15$ subjects, $p=0.95$ ), indicating that both conditions were perceived as similarly unintelligible.

\section{Distinct patterns of neurophysiological power changes as a result of perceptual pop-out}

Event-related power enhancement showed significant effects for STS, MTG, and IFG (Wilcoxon signed rank test, $N=16$ subjects, $p<0.05$; cluster statistics were used to correct for multiple comparisons for all the tests in this section). Figure 3 shows how this measure varies across the whole sentence duration (1.5 s) across all locations and frequency bands of interest. Sustained $\Delta$ band power enhancement was measured in left STS $(p<0.05)$ and left MTG $(p<0.05)$. MTG showed significant left lateralization of such enhancement (paired Wilcoxon signed rank test, $N=$ $16, p<0.05)$. Importantly, these sustained effects did not emerge for other frequency bands. A different pattern of results was measured in IFG, which showed early ( 100-550 ms) left-lateralized suppression of cumulative power in $\gamma$-band $(p<0.05$; significant left lateralization, paired Wilcoxon signed rank test, $N=16, p<0.05$ ) and right-biased enhancements for longer latencies for broadband power ( 600-1300 ms respectively; $p<0.05$; significant right lateralization emerged for the broadband signal: paired Wilcoxon signed rank test, $N=16, p<0.05$ ).

This pattern of enhancement and suppression of cortical activity, as depicted in Figure 3, is based on the 'interaction contrast' measure [(Pop-outpost - Pop-outpre) - (Unintelligiblepost Unintelligiblepre)], which isolates the effects of perceptual pop-out (Millman et al., 2015). Therefore, the present experiment successfully elicited an increase in perceived intelligibility that was reflected in the source-space MEG signal. In particular, the sustained effects in left STS and left MTG suggest that the perceptual change in speech intelligibility is underpinned by enhanced $\Delta$ band activity in those cortical locations. Shorter suppressive effects also emerged in HG and IFG. Although this result indicates that the MEG data reflects some effects of prior knowledge, this specific measure was insufficient to assess top-down/bottom-up cortical interactions and, importantly, it did not show overwhelming suppressive effects due to prior knowledge that would have supported other studies based on similar pop-out paradigms (Sohoglu et al., 2012; Blank and Davis, 2016; Sohoglu and Davis, 2016; Di Liberto et al., in review). One reason may lie in the event-related approach itself, which is ill-suited for the relatively long sentences used in this experiment (Crosse et al., 2016b): The fast dynamics of conversational speech hamper the investigation of long latency responses, as they overlap with the early responses to subsequent sounds. This is not the case when short isolated stimuli are used such as single words (Sohoglu et al., 2012; Sohoglu and Davis, 2016). For this reason, additional analyses were used that aimed at eliciting more targeted indices of cortical activity with the goal of determining the precise 
spatiotemporal dynamics due to the effect of prior knowledge.

Prior knowledge modulates top-down and bottom-up envelope entrainment

Cortical entrainment was used to determine how accurately the broadband envelope of speech could be reconstructed from the source-space MEG signals of individual participants, as measured by correlation (Pearson's $r$; Figures $\mathbf{S 1}$ and $\mathbf{S 2}$ report the results for individual conditions in $\Delta$ - and $\theta$ bands respectively). A change in this correlation when Unintelligiblepost was compared with Popoutpost (block 2) was used to quantify changes in cortical entrainment due to perceptual pop-out (see Figures S3 and S4). In addition, the potential contribution of physical differences between stimuli was derived by calculating the change in correlation between Unintelligiblepre and Pop-outpre (block 1) (see Figures S3 and S4). A repeated measures three-way ANOVA (with factors block, cortical location, and time-lag window) indicated a main effect of block in the $\Delta$-band $(F(1,15)=7.45 ; p=$ $\left.0.016 ; \eta^{2}=0.33\right)$ but not for $\theta$-band $\left(F(1,15)=2.17 ; p=0.162 ; \eta^{2}=0.13\right)$. In addition, the enhancements in cortical tracking due to prior knowledge was studied explicitly at every location of interest and time-lag window by subtracting the contrasts for the two blocks (block 2 - block 1), namely the 'interaction contrast' measure (Figure 4). A significant enhancement in cortical tracking involving both temporal and frontal sites emerged for the $\Delta$-band $(1-4 \mathrm{~Hz})$ at different latencies. Specifically, an early sustained enhancement of left IFG cortical areas arose from the interval -50$150 \mathrm{~ms}$, followed by a sustained enhancement of left HG beginning for the interval 0-200 ms (Wilcoxon signed rank test, $N=16, p<0.05$; correction for multiple comparisons was performed using cluster statistics that takes into account dependencies across the time dimension). The enhancement in both IFG and HG areas was sustained until the lag-window 150-350 ms. No significant effects emerged for the 'interaction contrast' measure in the $\theta$-band. These results establish a link between perceptual pop-out and increased cortical tracking of the speech envelope and, crucially, suggests that $\Delta$-band cortical activity in left IFG may initiate the early enhancement of envelope tracking enhancement in left HG.

The link between these results and the perceptual pop-out effect was controlled for by using the 'interaction contrast' measure, which isolates the effect of prior knowledge by controlling for the potential effect of physical differences between stimuli. Nevertheless, further insights can be drawn by considering the results in the $\Delta$-band (Figure S3) and $\theta$-band (Figure S4) for the two blocks separately. In block 1, any differences in the reconstruction accuracies between Unintelligiblepre and Pop-outpre represent a baseline that accounts for physical differences between the two vocoded sentences that do not involve the pop-out effect. Because the stimuli have similar spectro-temporal properties and were both perceived as unintelligible, it was predicted that no differences would have arisen for the contrast within block 1 . As expected, no significant change in cortical entrainment emerged for any time-lag window in both $\Delta$ - and $\theta$-bands.

As no effects were predicted for block 1, we expected block 2 to produce the same pattern of results that emerged for the 'interaction contrast'. The result partially confirmed our expectation for $\Delta$-band (Figure S3): A significant increase in cortical tracking emerged in block 2 for almost all the cortical areas of interest for the $\Delta$-band at different latencies, including the significance patterns that emerged for the 'interaction contrast' measure. Specifically, an early sustained enhancement of left cortical areas arose, starting from consecutive time-lag windows for, in this order, IFG, HG, STS, and MTG, from -50-150 ms, 0-200 ms, 50-250 ms, 100-300 ms respectively (Wilcoxon signedrank test, $N=16, p<0.05$; correction for multiple comparisons was performed using cluster statistics that takes into account dependencies across the time dimension). This suggests that the early activation of left IFG may initiate the early propagation of envelope entrainment within the 
left-hemisphere in a bottom-up direction from HG, to STS, and finally to MTG. A different pattern emerged for the envelope entrainment in the $\theta$-band (4-8 Hz), which showed an early suppression with perceptual enhancement in all cortical areas of interest. However, statistical significance emerged only for the suppressive effect in right IFG (Figure S4).

These findings provide detailed information on the effects of prior knowledge on the early cortical dynamics underlying continuous speech processing. In particular, they indicate that the availability of higher-level information in the upcoming stimulus, which enhances the perceived intelligibility of the speech sentences, increases the early $\Delta$-band tracking of the envelope of speech in left IFG. Enhanced $\Delta$-band entrainment was also measured in other areas in left temporal cortex at progressively longer latencies, suggesting that information flow initiated by left IFG then propagates to primary auditory cortex. The analysis of block 2 in isolation suggested that this might also be followed by effects in superior temporal and posterior middle temporal areas within the left hemisphere.

\section{Effects of prior knowledge on cortico-cortical dynamics}

The cortical entrainment analysis provided insights on how perceptual pop-out affects the encoding of the speech sentences measured with MEG. However, this approach is constrained to the speech features chosen for the analysis, which in this case consist of the speech envelope. Furthermore, while both the entrainment and power analyses study the effects of perceived intelligibility in each individual cortical area separately from the others, there are approaches that allow the explicit investigation of the causal interaction between cortical areas. Importantly, these approaches enable the study of how the pop-out effect modifies the spatiotemporal dynamics of the speech comprehension network, without biasing the analysis to specific features of speech (e.g. speech envelope). Therefore, further analysis was conducted with the goal of obtaining complementary insights on the cortical mechanisms of integration of prior information during speech comprehension.

Here, this was achieved by estimating effective connectivity within the eight-node network of locations of interest (bilateral HG, STS, MTG, and IFG) using a dDTF measure, which can be considered a conditional Granger causality measure that quantifies the influence of one time series on another time series, even in the presence of a third time series. In the context of a hierarchical structure of the speech processing network, we hypothesized that this measure would have shown interactions primarily between functionally neighboring stages, such as HG-STS and STS-MTG (e.g. Hickok and Poeppel, 2007), while based on previous studies, we had less defined predictions from the literature on what causal links might exist between IFG and temporal areas. Specifically, the dDTF analyses explicitly tested the following predictions based on previous work that used prior knowledge to modulate speech intelligibility: i) The emergence of top-down connections from IFG to STG/STS and of bottom-up connections from STG/STS to IFG (Sohoglu and Davis, 2016) and ii) top-down effects from STG to HG (Tuennerhoff and Noppeney, 2016). Furthermore, a broad literature suggests that hemispheric differences exist in the processing of sounds and speech (Poeppel, 2003; Gross et al., 2013; Peelle et al., 2013; Zoefel and VanRullen, 2016). For this reason, we predicted that most interactions would have emerged within, rather than between, hemispheres.

These hypotheses are partially reflected by the results in Figure 5. The space-frequency grid shows significant changes in bottom-up and top-down connectivity between cortical locations of interest due to perceptual pop-out. Positive values indicate a connectivity enhancement due to prior knowledge (from block 1 to block 2), while negative values reflect a connectivity suppression. 
Strong effects of prior information emerged as a suppression of the bottom-up link between left HG-left STS and as enhancement of the bottom-up link between left STS-left MTG. Opposite dynamics emerged for the corresponding top-down links, which exhibited a strong enhancement between left STS-left HG and a less prominent suppression between left MTG-left STS. Both these bottom-up and top-down effects involved most of the cortical frequencies of interest. Consistent but less prominent effects emerged for links originating from left IFG, which showed enhanced topdown connections with all the left temporal areas of interest: Granger causal links with left HG involved cortical frequencies above $15 \mathrm{~Hz}$ while links with left STS and left MTG involved slower cortical rhythms $(<15 \mathrm{~Hz})$. Furthermore, a strong inter-hemispheric interaction emerged for the connection from right $\mathrm{HG}$ to left $\mathrm{HG}$ across a large set of frequencies $(\sim 4-35 \mathrm{~Hz})$. In contrast, less consistent and prominent results emerged for connections within the right hemisphere, which exhibited effects of prior knowledge almost exclusively for bottom-up connections and slow cortical rhythms $(<15 \mathrm{~Hz})$. Prior knowledge strengthened connectivity between areas in the right temporal areas. Specifically, increased connectivity was measured between right HG and right STS (in both directions) and from right HG to right MTG. Secondly, the perceptual pop-out reduced Granger causal connections from various cortical areas (i.e., right HG, left and right MTG) to right IFG, with the only exception being the link from right STS to right IFG, which became stronger as 
The cortical mechanisms underpinning the integration of prior knowledge with sensory input during continuous speech comprehension are poorly understood. Here, we demonstrated that non-invasive MEG measures are sensitive to the predictive effects of prior knowledge on perceived speech intelligibility. Furthermore, we provided insight into the cortical spatiotemporal dynamics that underlie this process, and the implications for current views of the cortical underpinnings of speech comprehension.

\section{Low-frequency envelope entrainment reflects perceived speech intelligibility}

Measures of cortical entrainment to speech features, in particular to the speech envelope, are powerful tools to investigate the cortical mechanisms of continuous speech processing (Ahissar et al., 2001; Aiken and Picton, 2008; Nourski et al., 2009; O'Sullivan et al., 2014; Crosse et al., 2016a). However, it remains unclear to what extent envelope entrainment reflects the encoding of sensory information that is specific to speech (Ding and Simon, 2014b; Ding and Simon, 2014a; Di Liberto et al., 2015; Zoefel and VanRullen, 2016) and how entrainment is affected by higher-order processes such as the integration of prior knowledge (Holdgraf et al., 2016). It has been argued that speech intelligibility impacts on the phase of ongoing neural oscillations in (left) temporal cortex, which was interpreted as suggesting that linguistic information affects neural oscillations (Peelle et al., 2013; Park et al., 2015). Yet, those studies did not disentangle the effects of perception from physical (acoustic) differences, as the intelligibility was modulated using physical manipulations of the speech stimuli (e.g. noise-vocoding, time-reversing). A previous analysis of the same MEG dataset used in the present study did not reveal any significant effect of prior knowledge on phaselocking to the speech envelope (Millman et al., 2015), quantified using $\theta$-band coherence and crosscorrelation measures between stimulus envelope and source-space neural signals. The reason may have been methodological as cross-correlation is sub-optimal when studying responses to stimuli with speech-like statistics (Crosse et al., 2016b) and did not allow for an exploration of the temporal dynamics of the pop-out effect. A recent study that used an analysis framework aimed at relating ongoing EEG to particular features of a speech stimulus found a positive correlation between $\Delta$ band entrainment to phoneme-level features and perceived speech intelligibility using a pop-out paradigm similar to the one employed here (Di Liberto et al., in review). However, no significant effect of prior knowledge emerged specifically for envelope entrainment. It may be the case that the effect of prior knowledge on cortical entrainment is so subtle - relative to the entrainment to the acoustic energy of the stimulus itself - that the use of an imaging modality with higher spatial resolution than sensor-space EEG, combined with a stronger focus on time-domain analyses, is required to reveal this effect.

In contrast to our previous work (Millman et al., 2015) the results shown in Figure 4 clearly indicate that low-frequency envelope entrainment is affected by perceptual pop-out. Importantly, this effect was related specifically to the increase in perceived speech intelligibility and not to an effect of stimulus repetition or perceptual learning, as previously shown by Sohoglu and Davis (2016). Furthermore, the result was not a consequence of physical differences between the two stimuli as no effects on envelope entrainment were observed for block 1, in which both stimuli (Pop-out, Unintelligible) were unintelligible. Indeed, this finding is in line with the notion that cortical entrainment to the envelope of speech is prominent in the $\Delta$ - and $\theta$-bands (Ahissar et al., 2001; Aiken and Picton, 2008; Giraud and Poeppel, 2012; Gross et al., 2013; Keitel et al., 2017). Importantly, our results are in line with recent view suggesting that slow cortical activity reflects the effect of perceptual expectation (Arnal et al., 2011). In particular, low-frequency oscillations have been suggested to reflect multiple aspects of speech processing that previous research could not disambiguate. One possible solution to disentangling these myriad processes may be to investigate 
the time-locking to such low rhythms (Sedley et al., 2016). Here, we demonstrated the link between slow cortical oscillations and perceptual expectation specifically in the context of the envelope entrainment mechanism. Our results indicate that $\Delta$-band entrainment is strongly linked with speech intelligibility (Ding and Simon, 2013; Ding et al., 2014; Ding and Simon, 2014a) and with the formation of temporal predictions (Arnal et al., 2015), while $\theta$-band entrainment has been associated with the acoustic properties of the speech envelope (Ding and Simon, 2013; Peelle et al., 2013). Our results suggest a main role of $\Delta$-band entrainment in the predictive mechanisms that support an increase in the perceived intelligibility of speech.

Although these considerations confirm that envelope entrainment is modulated by perceptual popout, the specific underlying neural processes remains unclear. A recent fMRI study (Blank and Davis, 2016) reported that an increase in perceived speech clarity enhanced the encoding of speechspecific information in left STS, whilst the overall activation of left STS was suppressed. Such effects might be linked to the results presented in the current study, namely the $\Delta$-band enhancement (which showed spatiotemporal variability over time-lag windows from -50 to $400 \mathrm{~ms}$ ) and $\theta$-band suppression. In particular, the $\Delta$-band enhancement measured for STS could reflect the more detailed cortical encoding of speech-specific information described by Blank and Davis (2016). This hypothesis finds further support in the recent finding that prior knowledge, rather than simply producing increases or decreases in activation in specific cortical areas, enhances high $\gamma$-band entrainment specifically to speech-like spectrotemporal features in temporal cortex (Holdgraf et al., 2016). In line with the work from Blank and Davis (2016), and from Holdgraf et al. (2016), we contend that the $\Delta$-band enhancement measured in the present study may reflect similar sharpening mechanisms that enhance the cortical encoding of speech-like information. Similarly, the suppression in $\theta$-band may indicate an early reduced encoding of lower-level predictable acoustic features. Thus, a potential interpretation of these results in the context of predictive coding is that low-frequency envelope entrainment may reflect activity from both representational and error units (Friston and Kiebel, 2009; Clark, 2013).

\section{Top-down and bottom-up effects of prior knowledge during speech perception}

Speech comprehension is thought to involve the active integration of prior knowledge with sensory input through predictive top-down cortical mechanisms (Davis and Johnsrude, 2007; Wild et al., 2012; Blank and Davis, 2016; Leonard et al., 2016; Tuennerhoff and Noppeney, 2016; Di Liberto et al., in review), however the exact spatiotemporal dynamics of this process remain unclear. In particular, there is strong evidence that such effects occur in a broad network of cortical areas, including regions in temporal cortex and frontal areas such as IFG (Sohoglu et al., 2012; Park et al., 2015; Sohoglu and Davis, 2016). Our results advance the current understanding of this cortical network by providing new insights on the precise spatiotemporal dynamics of this process.

Here, we show that $\Delta$-band cortical entrainment to the envelope of speech is affected by prior knowledge, and that this effect rapidly propagates in a top-down manner, from left IFG to left HG (time windows $[-50,150] \mathrm{ms}$ and $[0,200] \mathrm{ms}$ respectively; Figure 4), and only subsequently reaches STS and then MTG (Figure S3). Interestingly, such a top-down phenomenon did not emerge for $\theta$-band (Figure S4), which instead showed suppressed envelope entrainment. This finding supports the notion that neural oscillations at different rates may contribute in distinct ways to predictive mechanisms in speech comprehension (Gross et al., 2013; Ding and Simon, 2014b; Fontolan et al., 2014; Kösem and van Wassenhove, 2016). In line with previous studies on speech comprehension, the early cortical entrainment measured in IFG indicates the involvement of a broader network that includes temporal and frontal sites (Hickok and Poeppel, 2007; Obleser and Kotz, 2009; Park et al., 2015; Sohoglu and Davis, 2016). Additionally, our results demonstrate that IFG exerts influence on regions in temporal cortex, supporting speech intelligibility through 
entrainment to the envelope of speech.

The cortical entrainment analysis indicated that the effects of prior knowledge entailed a rapid topdown propagation that affected all left cortical areas of interest between the time windows [-50, $150] \mathrm{ms}$ and $[100,300] \mathrm{ms}$. This result suggests that the effects of prior knowledge occur on a much shorter temporal scale than previously reported by similar pop-out studies (Sohoglu et al., 2012; Sohoglu and Davis, 2016). One explanation may be found in the use of short sentences in the present experiment. In contrast to isolated words, our stimuli are suitable for deriving entrainment measures in addition to event-related responses. Moreover, meaningful sentences enable the study of cortical responses to linguistic features. For these reasons, the present work highlights the importance of investigating both the cortical responses to isolated speech units and to continuous speech stimuli. Despite these advantages, it is possible that the use of the specific popout paradigm used by Millman et al. (2015) may explain some discrepancies with prior literature. One of the limitations of the experimental design used by Millman et al. (2015) was the repeated presentations of the speech sentences used for the conditions of interest i.e., Pop-out and Unintelligible. It is possible that repeated presentations of the same speech sentences could explain why the results of interest emerged mainly in $\Delta$-band: Previous work (Arnal et al., 2011) suggested that predictable speech stimuli result in distributed $\Delta$-band activity. Arnal et al. (2011) also found that $\beta$ and $\gamma$ oscillations index prediction error and its resolution. For this reason, the design of the specific popout paradigm used in the present study may be ill-suited to identifying neural representations of prediction error.

The cortical entrainment analysis provided a detailed picture of the top-down/bottom-up effects of prior knowledge in the speech comprehension network. However, the quantitative measures were biased toward a specific feature of speech: the temporal envelope. Furthermore, the order in which cortical entrainment emerges in different areas does not imply causality. This motivated further analysis of the MEG responses to unveil causal dynamics to which linear mapping between stimulus and neural recording would be insensitive. A connectivity analysis was performed on the source-space MEG signals to determine effective (directional) direct causality between the cortical regions of interest (Figure 5). It is important to clarify that our estimate of causality is constrained by two factors: 1) It is specific to dDTF measures, which can be thought as in the same domain of Granger causality (Blinowska, 2011) and 2) it is limited to the 8 cortical sites of interest. This analysis showed that perceptual pop-out increases the top-down (and suppresses the bottom-up) information flow between left HG and left STS, which is in line with the recent fMRI work (Tuennerhoff and Noppeney, 2016). Furthermore, the results in Figures 4, 5, and S3 provide new insights on the top-down link between IFG and STG that was previously reported by Blank and Davis (2016). Specifically, the effect of prior knowledge on the envelope entrainment that initiates in IFG propagates firstly to HG, and only subsequently to STS and MTG. In addition, direct Granger causal top-down links emerged between left IFG and all the left temporal sites of interest, indicating the direct influence of left IFG onto each of those temporal areas. Taken together, these results are compatible with theories of predictive coding which would explain such effects as an early top-down modulation, which increases the readiness of HG to process the upcoming stimulus, and the subsequent reduction of the bottom-up prediction error (Friston and Kiebel, 2009; Clark, 2013). A second effect was the enhancement of the bottom-up directional link from left STS to left posterior MTG. The use of short meaningful sentence stimuli (rather than isolated words) may be crucial for the interpretation of this result; in fact, such stimuli enable processing at the syntactic and semantic levels. In this context, previous research suggested the emergence of both activity suppression, at hierarchical levels where speech information is predictable, and activity enhancement, at levels that were previously not (or less) active (George et al., 1999; Tuennerhoff and Noppeney, 2016). Hence, our results indicate that posterior MTG is strongly involved in the processing of higher-level features of speech related to intelligibility (Lau et al., 2008; Turken and Dronkers, 2011; Henseler et al., 2014; Zhang et al., 2015; Tuennerhoff and Noppeney, 2016). The 
focus on local interactions was essential to reveal the various pattern of this "vertical" information

Finally, our results suggest the involvement of a broader network of cortical areas than previously modeled (Sohoglu and Davis, 2016; Tuennerhoff and Noppeney, 2016) for the predictive effect of prior information, including left frontal and temporal cortices. In line with previous studies, we contend that early integration of prior knowledge in IFG enhances the readiness of the network to the expected incoming stimulus in lower-order temporal areas (Friston, 2005; Sohoglu and Davis, 2016). Hence, frontal areas constitute a crucial part of such a cortical network (c.f. Tuennerhoff and Noppeney, 2016). Furthermore, our results indicate that multiple temporal sites should be included when modeling the interactions between frontal and temporal cortex during perceptual prediction. In fact, IFG may interact differently with distinct temporal areas, which could not be captured by a simpler two-node model (Sohoglu and Davis, 2016).

In summary, we provided detailed spatiotemporal evidence of the top-down and bottom-up dynamics of prior knowledge on speech intelligibility using a pop-out paradigm. First, our results indicate that non-invasive measures of envelope entrainment are sensitive to the predictive effect of prior knowledge. Second, prior knowledge induces rapid information flow in $\Delta$-band cortical signals that initiates in left IFG and subsequently propagates from HG to STS and finally to MTG in the left hemisphere. This indicates that left IFG may induce rapid $(<50 \mathrm{~ms})$ top-down modulation of lower levels of the speech processing hierarchy. In line with the notion of predictive coding, an effective connectivity analysis revealed that perception of intelligible speech sentences increased top-down information flow from left STS to left HG and enhanced the bottom-up flow from left STS to left MTG, suggesting the involvement of left posterior MTG in the processing of intelligible speech features.

\section{Acknowledgements}

This study was supported by an Irish Research Council Government (GOIPG, 2013-2017) of Ireland Postgraduate Scholarship and by a travel grant from Guarantors of Brain (UK registered charity). The authors thank Bahman Nasseroleslami for useful discussions on the connectivity analysis approach.

\section{References}

Ahissar E, Nagarajan S, Ahissar M, Protopapas A, Mahncke H, Merzenich MM (2001) Speech comprehension is correlated with temporal response patterns recorded from auditory cortex. Proceedings of the National Academy of Sciences 98:13367-13372.

Aiken SJ, Picton TW (2008) Human cortical responses to the speech envelope. Ear Hear 29:139157.

Arnal LH, Doelling KB, Poeppel D (2015) Delta-Beta Coupled Oscillations Underlie Temporal Prediction Accuracy. Cerebral Cortex (New York, NY) 25:3077-3085.

Arnal LH, Wyart V, Giraud AL (2011) Transitions in neural oscillations reflect prediction errors generated in audiovisual speech. Nat. Neurosci., 14, pp. 797-801.

Blank H, Davis MH (2016) Prediction Errors but Not Sharpened Signals Simulate Multivoxel fMRI Patterns during Speech Perception. PLoS Biol 14:e1002577.

Blinowska KJ (2011) Review of the methods of determination of directed connectivity from multichannel data. Medical \& Biological Engineering \& Computing 49:521-529.

Bornkessel-Schlesewsky I, Schlesewsky M, Small SL, Rauschecker JP (2015) Neurobiological roots of language in primate audition: common computational properties. Trends in cognitive sciences 19:142-150. 
Chang EF, Rieger JW, Johnson K, Berger MS, Barbaro NM, Knight RT (2010) Categorical speech representation in human superior temporal gyrus. Nat Neurosci 13:1428-1432.

Clark A (2013) Whatever next? Predictive brains, situated agents, and the future of cognitive science. The Behavioral and brain sciences 36:181-204.

Crosse MJ, Di Liberto GM, Lalor EC (2016a) Eye Can Hear Clearly Now: Inverse Effectiveness in Natural Audiovisual Speech Processing Relies on Long-Term Crossmodal Temporal Integration. J Neurosci 36:9888-9895.

Crosse MJ, Di Liberto GM, Bednar A, Lalor EC (2016b) The Multivariate Temporal Response Function (mTRF) Toolbox: A MATLAB Toolbox for Relating Neural Signals to Continuous Stimuli. Frontiers in Human Neuroscience 10.

Davis MH, Johnsrude IS (2003) Hierarchical processing in spoken language comprehension. The Journal of neuroscience : the official journal of the Society for Neuroscience 23:3423-3431.

Davis MH, Johnsrude IS (2007) Hearing speech sounds: top-down influences on the interface between audition and speech perception. Hear Res 229:132-147.

Davis MH, Johnsrude IS, Hervais-Adelman A, Taylor K, McGettigan C (2005) Lexical information drives perceptual learning of distorted speech: evidence from the comprehension of noisevocoded sentences. Journal of Experimental Psychology: General 134:222.

Delorme A, Mullen T, Kothe C, Akalin Acar Z, Bigdely-Shamlo N, Vankov A, Makeig S (2011) EEGLAB, SIFT, NFT, BCILAB, and ERICA: New Tools for Advanced EEG Processing. Computational Intelligence and Neuroscience 2011:12.

DeWitt I, Rauschecker JP (2012) Phoneme and word recognition in the auditory ventral stream. Proceedings of the National Academy of Sciences of the United States of America 109:E505-514.

Di Liberto GM, O'Sullivan JA, Lalor EC (2015) Low-Frequency Cortical Entrainment to Speech Reflects Phoneme-Level Processing. Curr Biol 25:2457-2465.

Di Liberto GM, Crosse MJ, Lalor EC (in review) Cortical measures of phoneme-level speech encoding correlate with the perceived clarity of natural speech. JN-RM-0648-17.

Ding M, Chen Y, Bressler SL (2006) 17 Granger Causality: Basic Theory and Application to Neuroscience. Handbook of time series analysis: recent theoretical developments and applications:437.

Ding M, Bressler SL, Yang W, Liang H (2000) Short-window spectral analysis of cortical eventrelated potentials by adaptive multivariate autoregressive modeling: data preprocessing, model validation, and variability assessment. Biological Cybernetics 83:35-45.

Ding N, Simon JZ (2013) Adaptive temporal encoding leads to a background-insensitive cortical representation of speech. The Journal of neuroscience : the official journal of the Society for Neuroscience 33:5728-5735.

Ding N, Simon JZ (2014a) Cortical Entrainment to Continuous Speech: Functional Roles and Interpretations. Frontiers in Human Neuroscience 8.

Ding N, Simon JZ (2014b) Cortical entrainment to continuous speech: functional roles and interpretations. Front Hum Neurosci 8:311.

Ding N, Chatterjee M, Simon JZ (2014) Robust cortical entrainment to the speech envelope relies on the spectro-temporal fine structure. Neuroimage 88:41-46.

Dudley H (1939) Remaking Speech. The Journal of the Acoustical Society of America 11:169-177.

Evans AC, Collins DL, Mills SR, Brown ED, Kelly RL, Peters TM (1993) 3D statistical neuroanatomical models from 305 MRI volumes. In: 1993 IEEE Conference Record Nuclear Science Symposium and Medical Imaging Conference, pp 1813-1817 vol.1813.

Fontolan L, Morillon B, Liegeois-Chauvel C, Giraud AL (2014) The contribution of frequencyspecific activity to hierarchical information processing in the human auditory cortex. Nat Commun 5:4694.

Foster JR, Summerfield AQ, Marshall DH, Palmer L, Ball V, Rosen S (1993) Lip-reading the BKB sentence lists: Corrections for list and practice effects. British Journal of Audiology 27:233246. 
Friederici AD, Kotz SA, Scott SK, Obleser J (2010) Disentangling syntax and intelligibility in auditory language comprehension. Human brain mapping 31:448-457.

Friston K (2005) A theory of cortical responses. Philosophical transactions of the Royal Society of London Series B, Biological sciences 360:815-836.

Friston K, Kiebel S (2009) Predictive coding under the free-energy principle. Philosophical transactions of the Royal Society of London Series B, Biological sciences 364:1211-1221.

George N, Dolan RJ, Fink GR, Baylis GC, Russell C, Driver J (1999) Contrast polarity and face recognition in the human fusiform gyrus. Nat Neurosci 2:574-580.

Giraud AL, Poeppel D (2012) Cortical oscillations and speech processing: emerging computational principles and operations. Nat Neurosci 15:511-517.

Gow DW, Jr., Segawa JA (2009) Articulatory mediation of speech perception: a causal analysis of multi-modal imaging data. Cognition 110:222-236.

Granger CWJ (1969) Investigating Causal Relations by Econometric Models and Cross-spectral Methods. Econometrica 37:424-438.

Groppe DM, Urbach TP, Kutas M (2011) Mass univariate analysis of event-related brain potentials/fields I: A critical tutorial review. Psychophysiology 48:1711-1725.

Gross J, Hoogenboom N, Thut G, Schyns P, Panzeri S, Belin P, Garrod S (2013) Speech rhythms and multiplexed oscillatory sensory coding in the human brain. PLoS Biol 11:e1001752.

Henseler I, Mädebach A, Kotz SA, Jescheniak JD (2014) Modulating Brain Mechanisms Resolving Lexico-semantic Interference during Word Production: A Transcranial Direct Current Stimulation Study. Journal of cognitive neuroscience 26:1403-1417.

Hickok G, Poeppel D (2007) The cortical organization of speech processing. Nature reviews Neuroscience 8:393-402.

Holdgraf CR, de Heer W, Pasley B, Rieger J, Crone N, Lin JJ, Knight RT, Theunissen FE (2016) Rapid tuning shifts in human auditory cortex enhance speech intelligibility. Nat Commun 7:13654.

Huang MX, Mosher JC, Leahy RM (1999) A sensor-weighted overlapping-sphere head model and exhaustive head model comparison for MEG. Phys Med Biol 44:423-440.

Huang MX, Shih JJ, Lee RR, Harrington DL, Thoma RJ, Weisend MP, Hanlon F, Paulson KM, Li T, Martin K, Millers GA, Canive JM (2004) Commonalities and differences among vectorized beamformers in electromagnetic source imaging. Brain Topogr 16:139-158.

Humphries C, Sabri M, Lewis K, Liebenthal E (2014) Hierarchical organization of speech perception in human auditory cortex. Front Neurosci 8.

Ing A, Schwarzbauer C (2014) Cluster Size Statistic and Cluster Mass Statistic: Two Novel Methods for Identifying Changes in Functional Connectivity Between Groups or Conditions. PLOS ONE 9(6): e98697.

Johnson S, Prendergast G, Hymers M, Green G (2011) Examining the Effects of One- and ThreeDimensional Spatial Filtering Analyses in Magnetoencephalography. PLOS ONE 6:e22251.

Keitel A, Ince RAA, Gross J, Kayser C (2017) Auditory cortical delta-entrainment interacts with oscillatory power in multiple fronto-parietal networks. NeuroImage 147:32-42.

Korzeniewska A, Manczak M, Kaminski M, Blinowska KJ, Kasicki S (2003) Determination of information flow direction among brain structures by a modified directed transfer function (dDTF) method. Journal of neuroscience methods 125:195-207.

Kösem A, van Wassenhove V (2016) Distinct contributions of low- and high-frequency neural oscillations to speech comprehension. Language, Cognition and Neuroscience:1-9.

Kozinska D, Carducci F, Nowinski K (2001) Automatic alignment of EEG/MEG and MRI data sets. Clinical neurophysiology : official journal of the International Federation of Clinical Neurophysiology 112:1553-1561.

Kus R, Kaminski M, Blinowska KJ (2004) Determination of EEG activity propagation: pair-wise versus multichannel estimate. IEEE Trans Biomed Eng 51:1501-1510.

Lalor EC, Power AJ, Reilly RB, Foxe JJ (2009a) Resolving precise temporal processing properties of the auditory system using continuous stimuli. J Neurophysiol 102:349-359. 
Lalor EC, Power AJ, Reilly RB, Foxe JJ (2009b) Resolving Precise Temporal Processing Properties of the Auditory System Using Continuous Stimuli. J Neurophysiol 102:349-359.

Lau EF, Phillips C, Poeppel D (2008) A cortical network for semantics: (de)constructing the N400. Nature reviews Neuroscience 9:920-933.

Leonard MK, Baud MO, Sjerps MJ, Chang EF (2016) Perceptual restoration of masked speech in human cortex. Nature Communications 7:13619.

Lewis AG, Bastiaansen M (2015) A predictive coding framework for rapid neural dynamics during sentence-level language comprehension. Cortex 68:155-168.

Lütkepohl H (2007) New Introduction to Multiple Time Series Analysis: Springer Berlin Heidelberg.

Macleod A, Summerfield Q (1987) Quantifying the contribution of vision to speech perception in noise. British Journal of Audiology 21:131-141.

Maris E (2012) Statistical testing in electrophysiological studies. Psychophysiology 49:549-565.

Mesgarani N, Cheung C, Johnson K, Chang EF (2014) Phonetic Feature Encoding in Human Superior Temporal Gyrus. Science 343:1006-1010.

Millman RE, Johnson SR, Prendergast G (2015) The role of phase-locking to the temporal envelope of speech in auditory perception and speech intelligibility. Journal of cognitive neuroscience 27:533-545.

Millman RE, Prendergast G, Hymers M, Green GG (2013) Representations of the temporal envelope of sounds in human auditory cortex: can the results from invasive intracortical "depth" electrode recordings be replicated using non-invasive MEG "virtual electrodes"? NeuroImage 64:185-196.

Moore BCJ, Glasberg BR (1983) Suggested formulae for calculating auditory-filter bandwidths and excitation patterns. The Journal of the Acoustical Society of America 74:750-753.

Norris D, McQueen JM, Cutler A (2016) Prediction, Bayesian inference and feedback in speech recognition. Lang Cogn Neurosci 31:4-18.

Nourski KV, Reale RA, Oya H, Kawasaki H, Kovach CK, Chen H, Howard MA, 3rd, Brugge JF (2009) Temporal envelope of time-compressed speech represented in the human auditory cortex. The Journal of neuroscience : the official journal of the Society for Neuroscience 29:15564-15574.

O'Sullivan JA, Power AJ, Mesgarani N, Rajaram S, Foxe JJ, Shinn-Cunningham BG, Slaney M, Shamma SA, Lalor EC (2014) Attentional Selection in a Cocktail Party Environment Can Be Decoded from Single-Trial EEG. Cerebral Cortex:bht 355.

Obleser J (2014) Putting the Listening Brain in Context. Language and Linguistics Compass 8:646658.

Obleser J, Kotz SA (2009) Expectancy constraints in degraded speech modulate the language comprehension network. Cerebral Cortex:bhp128.

Overath T, McDermott JH, Zarate JM, Poeppel D (2015) The cortical analysis of speech-specific temporal structure revealed by responses to sound quilts. Nat Neurosci 18:903-911.

Park H, Ince RA, Schyns PG, Thut G, Gross J (2015) Frontal top-down signals increase coupling of auditory low-frequency oscillations to continuous speech in human listeners. Curr Biol 25:1649-1653.

Peelle JE, Johnsrude IS, Davis MH (2010) Hierarchical Processing for Speech in Human Auditory Cortex and Beyond. Frontiers in Human Neuroscience 4:51.

Peelle JE, Gross J, Davis MH (2013) Phase-locked responses to speech in human auditory cortex are enhanced during comprehension. Cerebral cortex (New York, NY : 1991) 23:1378-1387.

Poeppel D (2003) The analysis of speech in different temporal integration windows: cerebral lateralization as 'asymmetric sampling in time'. Speech communication 41:245-255.

Puvvada KC, Simon JZ (2017) Cortical Representations of Speech in a Multi-talker Auditory Scene. J Neurosci:0938-0917.

Rademacher J, Morosan P, Schormann T, Schleicher A, Werner C, Freund HJ, Zilles K (2001) Probabilistic mapping and volume measurement of human primary auditory cortex. 
NeuroImage 13:669-683.

Scott SK, Johnsrude IS (2003) The neuroanatomical and functional organization of speech perception. Trends in neurosciences 26:100-107.

Sedley W, Gander PE, Kumar S, Kovach CK, Oya H, Kawasaki H, Howard MA, Griffiths TD (2016) Neural signatures of perceptual inference Elife, 5.

Sohoglu E, Davis MH (2016) Perceptual learning of degraded speech by minimizing prediction error. Proceedings of the National Academy of Sciences of the United States of America 113:E1747-1756.

Sohoglu E, Peelle JE, Carlyon RP, Davis MH (2012) Predictive top-down integration of prior knowledge during speech perception. The Journal of neuroscience : the official journal of the Society for Neuroscience 32:8443-8453.

Stephan KE, Harrison LM, Kiebel SJ, David O, Penny WD, Friston KJ (2007) Dynamic causal models of neural system dynamics:current state and future extensions. J Biosci 32:129-144.

Tuennerhoff J, Noppeney U (2016) When sentences live up to your expectations. NeuroImage 124:641-653.

Turken AU, Dronkers NF (2011) The neural architecture of the language comprehension network: converging evidence from lesion and connectivity analyses. Frontiers in systems neuroscience 5:1.

Van Veen BD, van Drongelen W, Yuchtman M, Suzuki A (1997) Localization of brain electrical activity via linearly constrained minimum variance spatial filtering. IEEE Trans Biomed Eng 44:867-880.

Wild CJ, Davis MH, Johnsrude IS (2012) Human auditory cortex is sensitive to the perceived clarity of speech. NeuroImage 60:1490-1502.

Zhang L, Yue Q, Zhang Y, Shu H, Li P (2015) Task-dependent modulation of regions in the left temporal cortex during auditory sentence comprehension. Neuroscience Letters 584:351355.

Zoefel B, VanRullen R (2016) EEG oscillations entrain their phase to high-level features of speech sound. NeuroImage 124:16-23. 

(adapted from Millman et al., 2015). MEG data were recorded while participants listened to speech sentences that were degraded using 3-channel tone-carrier vocoded speech (Pop-outpre and

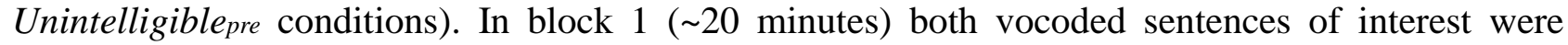
perceived as unintelligible. A training block followed ( $\sim 3$ minutes in which MEG data was not recorded) in which participants listened to the vocoded and the original versions of only one of the two sentences. Finally, both vocoded sentences of interest were presented in block 2 ( 20 minutes). In the latter case, the "pop-out" sentence (Pop-outpost condition) became intelligible after training, whereas the other sentence of interest remained unintelligible (Unintelligible post condition) because participants were not exposed to the corresponding original version.

Figure 2: Schematic of the cortical locations of interest. A standard MNI brain is used to display the 4 bilateral cortical areas selected for this study.

Figure 3: Perceptual "pop-out" determines changes in source-space MEG power. Event-related fields time-locked to sentence onsets were derived and power measures were calculated for each time sample from a latency of zero. Baseline correction was applied using the time interval from 0.5 to $-0.2 \mathrm{~ms}$. The cumulative difference in power ('interaction contrast': (Pop-outpost - Pop-outpre) - (Unintelligiblepost - Unintelligiblepre) ) is reported here for all frequency bands and cortical sources of interest (left and right hemispheres are directly compared to investigate possible asymmetries; values on the $y$-axis are reported in arbitrary units). Significant power changes and hemispheric asymmetries are marked with horizontal lines (Wilcoxon signed rank tests, $N=16, p<0.05$; cluster statistics across the time dimension were used to correct for multiple comparisons). Note that IFG and results for broadband signals are shown on different ordinate scales as they exhibited eventrelated power within a different range of magnitudes, compared with the other locations and frequency bands of interest.

Figure 4: Prior information induces top-down dynamics of $\Delta$-band entrainment to the speech envelope. TRFs were evaluated from the cortical responses to the envelope of the stimulus for a number of time-lag windows at progressively longer latencies (backward modeling approach; window size: $200 \mathrm{~ms}$ ). The quality of fit was estimated by calculating correlations between the speech envelope and its reconstructions using cross-validation. The perceptual pop-out effect is here shown by means of the 'interaction contrast' [(Pop-outpost - Pop-outpre) - (Unintelligiblepost Unintelligiblepre)], where only the Pop-outpost stimulus was perceived as intelligible. Results are reported for $\Delta$ - and $\theta$-bands and for each cortical location of interest. Significant effects represent cortical entrainment enhancement induced by prior information between Pop-out and Unintelligible sentences. Significant effects emerged for $\Delta$-band (Wilcoxon signed rank test, $N=16,{ }^{*} p<0.05$; cluster statistics across the time dimension were used to correct for multiple comparisons). Note that outlier values are reported separately from the correspondent distribution to improve the clarity of the figure, however they were included in the statistical analysis.

Figure 5: A source-space connectivity analysis: Low-level top-down and higher-level bottomup connections are enhanced when prior information is available.

Frequency grid showing the event-related grand-average $(N=16)$ change in brain dynamics induced by prior information across frequency, and cortical locations (SIFT; Delorme et al., 2011). dDTF (Korzeniewska et al., 2003) were calculated for an 8 node model, including all locations of interest. These can be interpreted as frequency-domain conditional Granger causality measures. Frequency grids show the dDTF contrast from block 1 to block 2, for Unintelligible (green) and Pop-out (orange) stimuli. Significant differences between stimulus type (i.e., the "interaction contrast') are highlighted with the grey shaded area (paired Wilcoxon signed rank test, $N=16, p<$ 0.05; Bonferroni correction was applied). 
Figure S1: Envelope reconstruction correlations for individual conditions in $\Delta$-band. TRFs were evaluated from the $\Delta$-band cortical responses to the envelope of the stimulus (backward modeling approach; window size: $200 \mathrm{~ms}$ ). The quality of fit was estimated by calculating correlations between the speech envelope and its reconstructions using cross-validation. This figure shows these correlation values for individual conditions.

Figure S2: Envelope reconstruction correlations for individual conditions in $\boldsymbol{\theta}$-band. TRFs were evaluated from the $\theta$-band cortical responses to the envelope of the stimulus (backward modeling approach; window size: 200ms). The quality of fit was estimated by calculating correlations between the speech envelope and its reconstructions using cross-validation. This figure shows these correlation values for individual conditions.

Figure S3: Effects of prior information on the $\Delta$-band entrainment to the speech envelope.

TRFs were evaluated from the cortical responses to the envelope of the stimulus (backward modeling approach; window size: 200ms). The quality of fit was estimated by calculating correlations between the speech envelope and its reconstructions using cross-validation. Differences between Pop-out and Unintelligible sentences were calculated for both blocks 1 and 2 (before and after exposure to the original sentence in the Pop-out condition). This procedure was repeated for each cortical location of interest. Significant effects emerged for $\Delta$-band (Wilcoxon signed rank test, $N=16, * p<0.05$; cluster statistics across the time dimension were used to correct for multiple comparisons). Differences between Pop-out and Unintelligible stimuli for each of the blocks are shown, where only the Pop-out stimulus was perceived as intelligible. Significant effects in block 2 represent cortical entrainment enhancement induced by prior information and physical differences between Pop-out and Unintelligible sentences. Significant contrasts in block 1 are caused by differences in entrainment brought about by physical differences between the stimuli. Note that outlier values are reported separately from the correspondent distribution to improve the clarity of the figure, however they were included in the statistical analysis.

Figure S4: Effects of prior information on the $\theta$-band entrainment to the speech envelope. TRFs were evaluated from the cortical responses to the envelope of the stimulus (backward modeling approach; window size: 200ms). The quality of fit was estimated by calculating correlations between the speech envelope and its reconstructions using cross-validation. Differences between Pop-out and Unintelligible sentences were calculated for both blocks 1 and 2 (before and after exposure to the original sentence in the Pop-out condition). This procedure was repeated for each cortical location of interest. Some significant effects were identified for $\theta$-bands (Wilcoxon signed rank test, $N=16,{ }^{*} p<0.05$; cluster statistics across the time dimension was used to correct for multiple comparisons). Note that outlier values are reported separately from the correspondent distribution to improve the clarity of the figure, however they were included in the statistical analysis.

\section{Figure S5: Source-space connectivity analyses reveal that low-level top-down and higher-level bottom-up connections are enhanced when prior information is available.}

Time-frequency grid showing the event-related grand-average $(\mathrm{N}=16)$ change in brain dynamics induced by prior knowledge across time, frequency, and cortical locations (SIFT; Delorme et al., 2011). Direct Directed Transfer Function measures (dDTF; Korzeniewska et al., 2003) were calculated for an 8-node model, including all locations of interest. These can be interpreted as frequency-domain conditional Granger causality measures. Time-frequency grids show the dDTF contrast from block 1 to block 2 for A) Unintelligible and B) Pop-out stimuli. The baseline is -100 to $-10 \mathrm{~ms}$ and the vertical red dashed lines indicates latency of the beginning of a sentence $(t=0$ $\mathrm{ms})$. The dDTF contrast measures were thresholded for statistical significance using the $95^{\text {th }}$ percentile of all measured values. 
Figure 1

Click here to download high resolution image

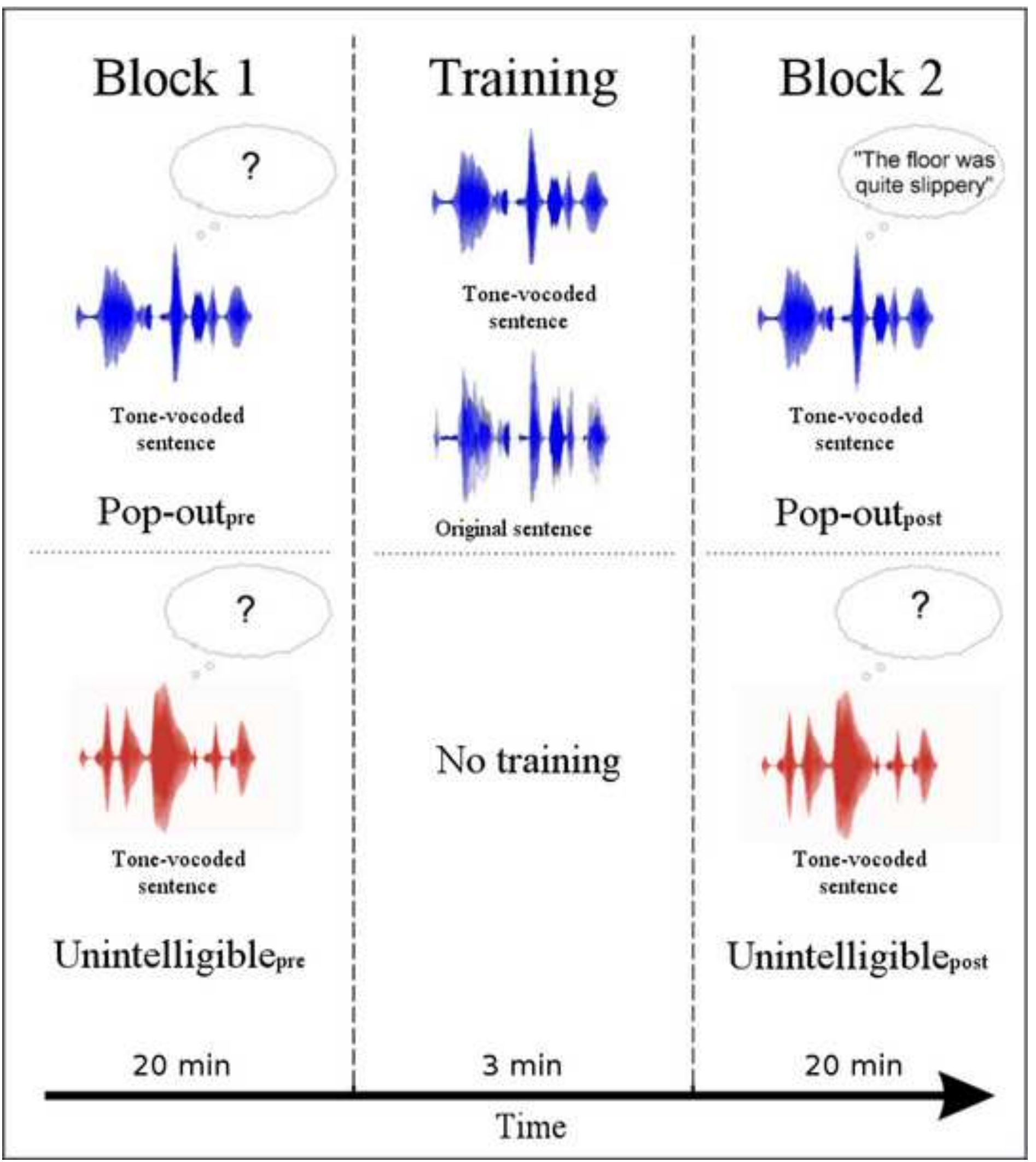

Time 

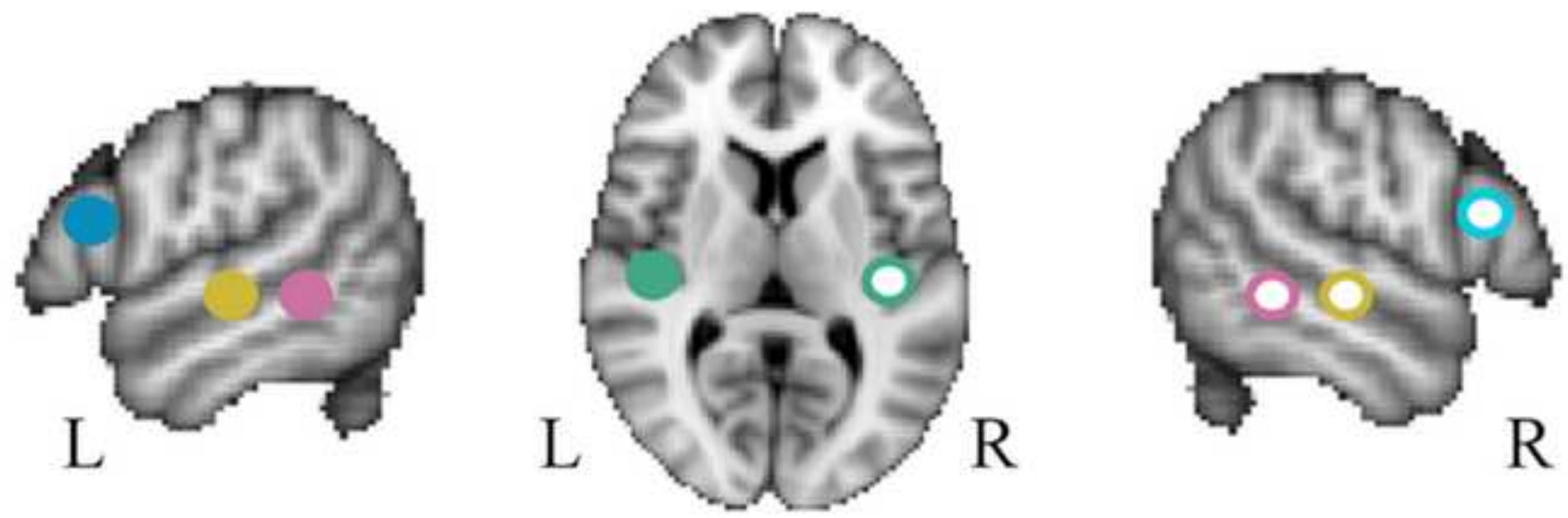

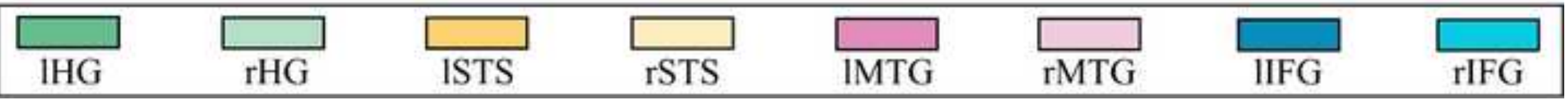




\section{Figure 3}

Click here to download high resolution image

\section{Cumulative sum of ERF Power enhancement}

(Pop-outpost - Pop-out $t_{\text {pre }}$ ) - (Unintpost - Unintpro)

HG

STS

MTG

IFG

$1-4 \mathrm{~Hz}$
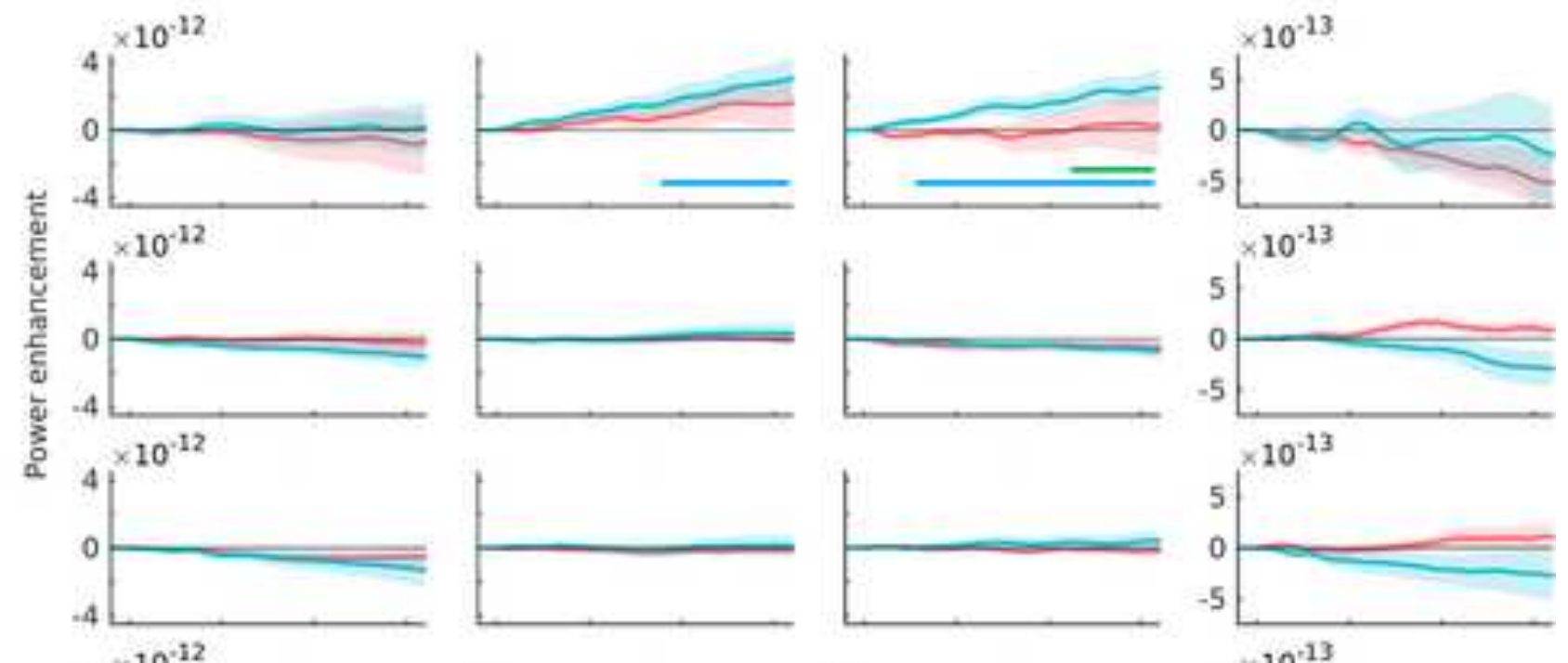

$4-8 \mathrm{~Hz}$

8-15 Hz
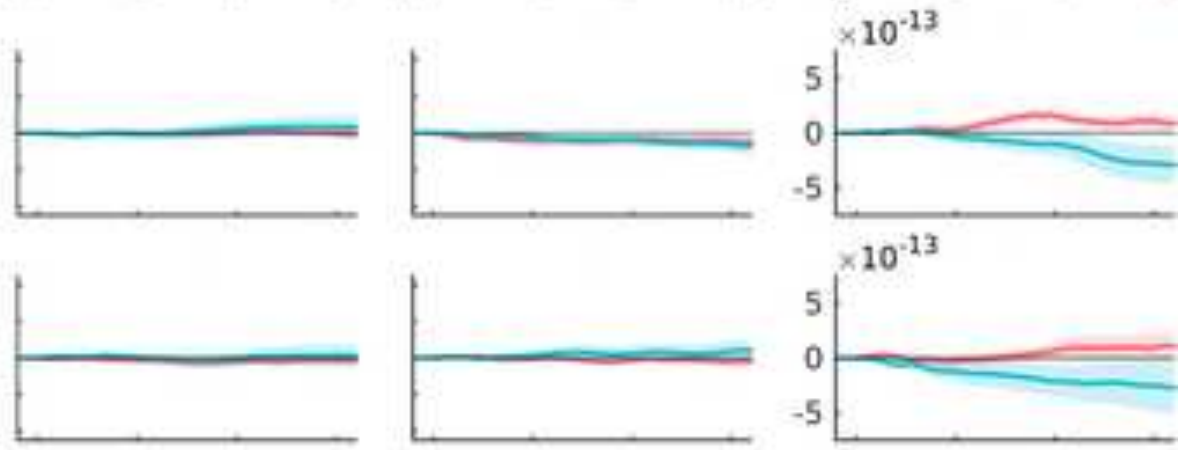

$15-30 \mathrm{~Hz}$
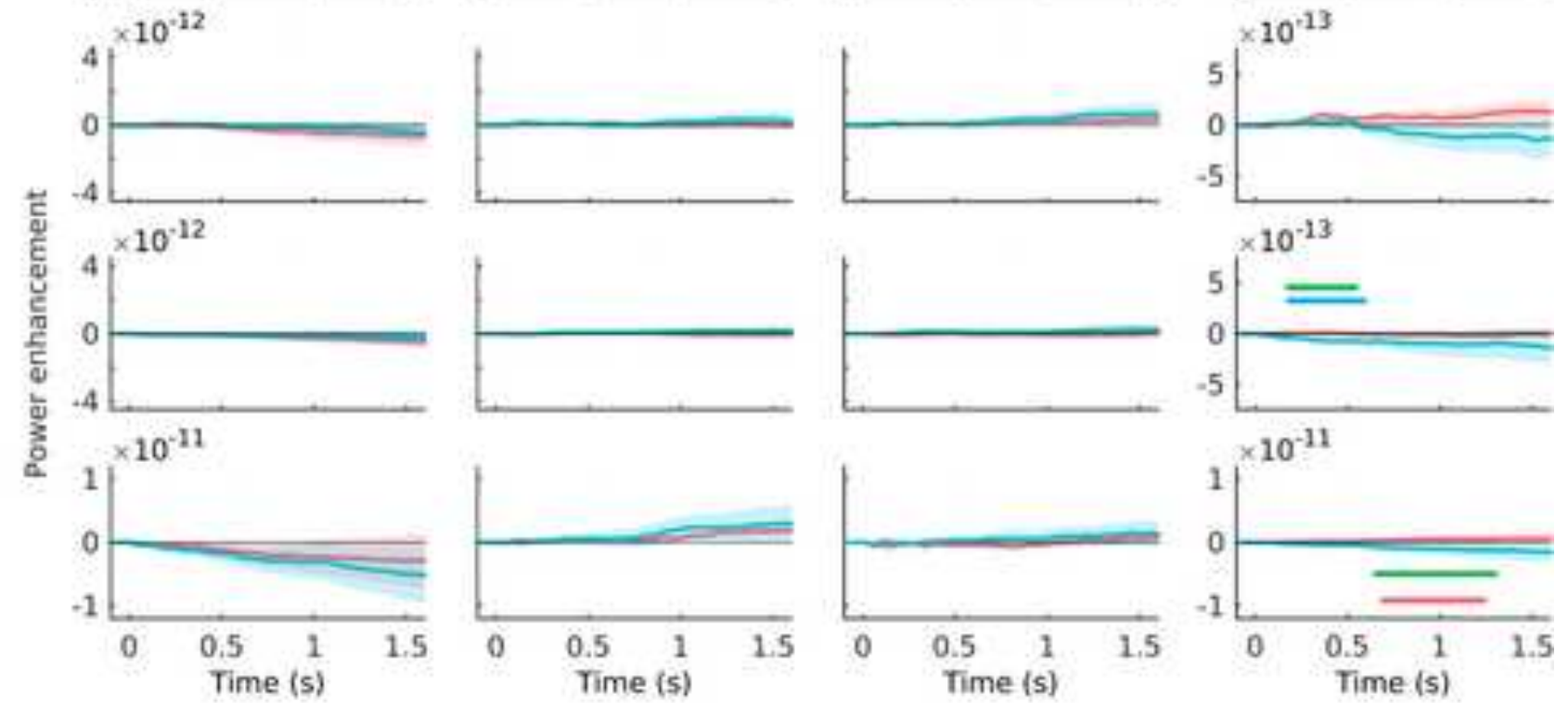

$30-45 \mathrm{~Hz}$

$1-45 \mathrm{~Hz}$
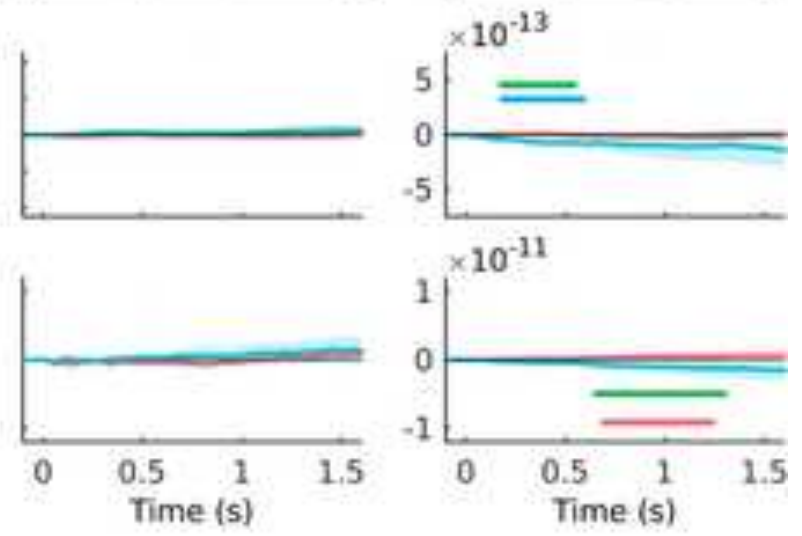

Hemisphere

Significance $(p<0.05)$

- Right

- Left

- Left hemisphere

- Asymmetry

- Right hemisphere (

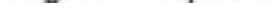


Interaction contrast

Delta-band
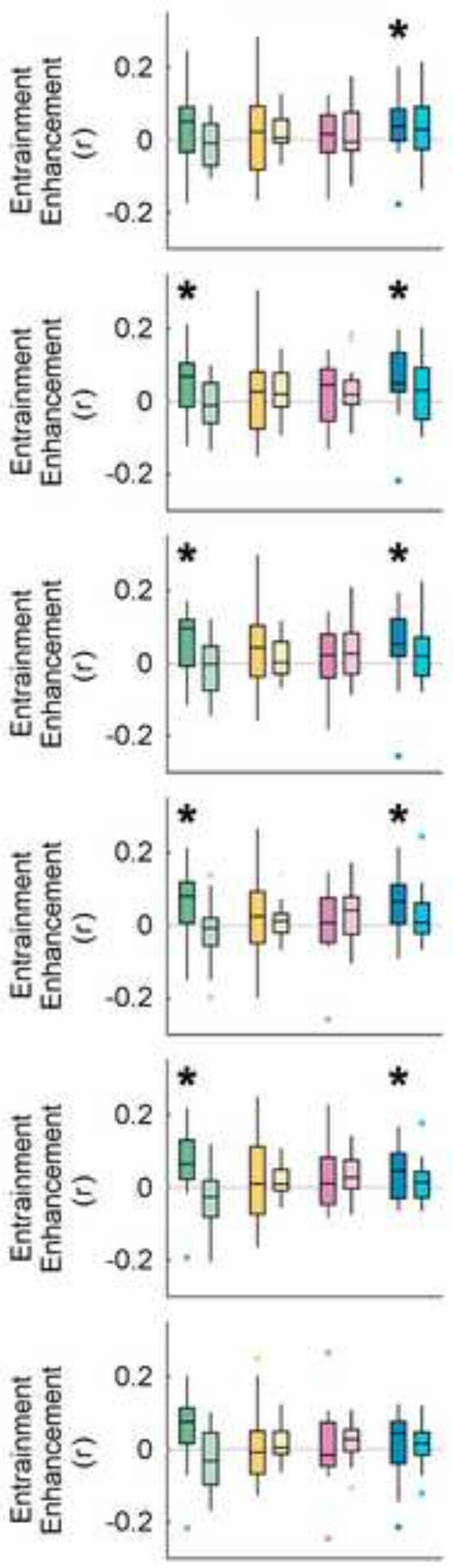

Theta-band

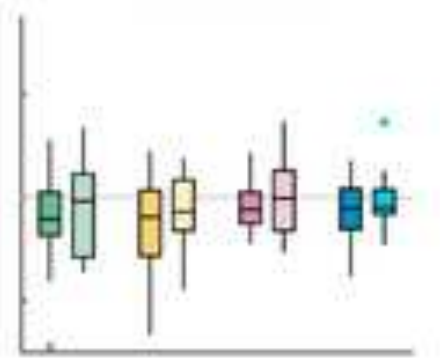

$-50-150 \mathrm{~ms}$

$0-200 \mathrm{~ms}$

$50-250 \mathrm{~ms}$

$100-300 \mathrm{~ms}$

$150-350 \mathrm{~ms}$

$200-400 \mathrm{~ms}$

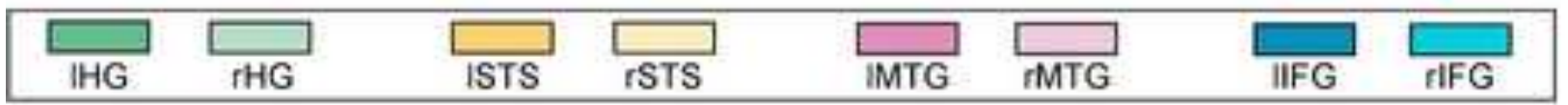




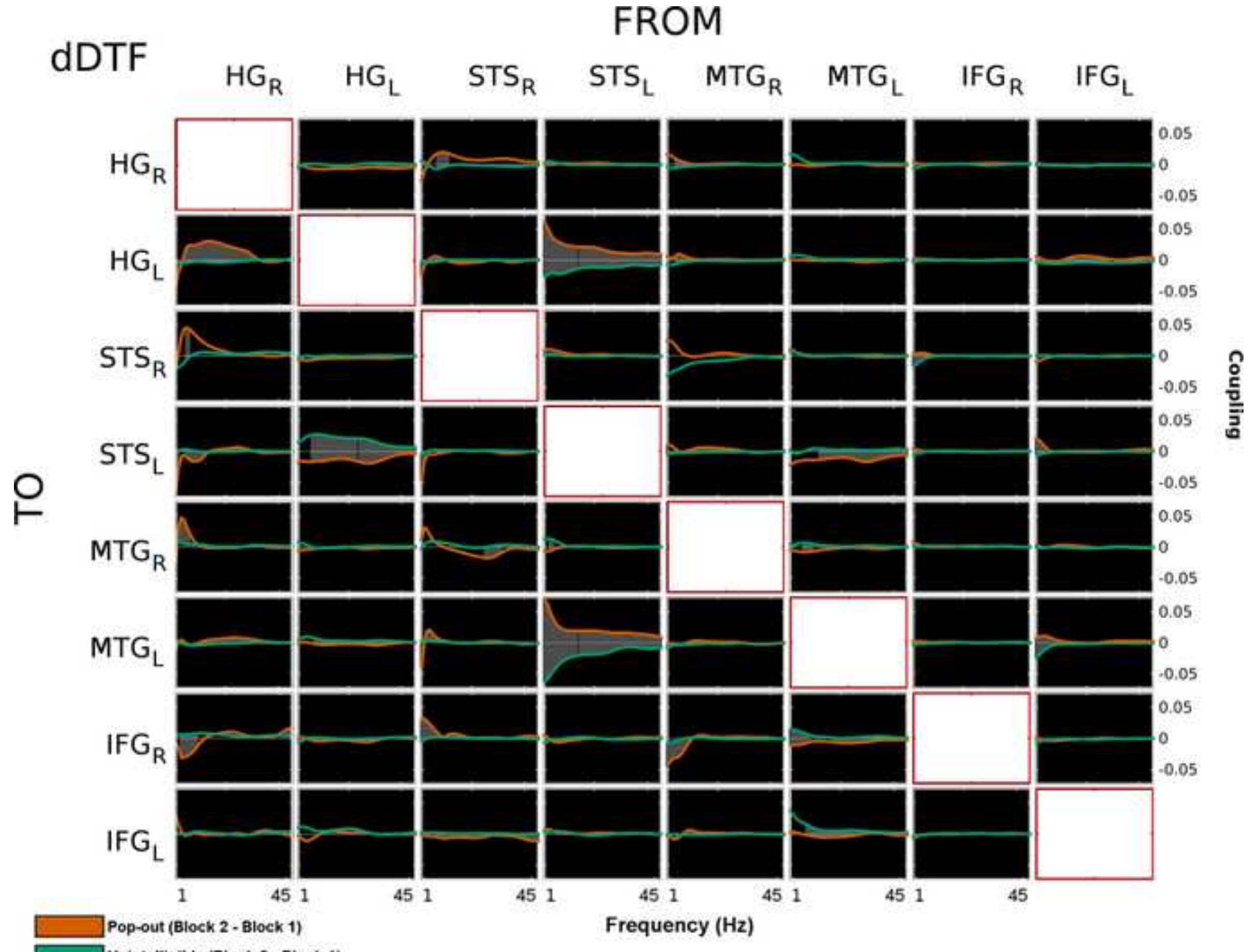

\section{to download high resolution image}

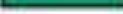

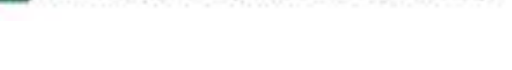

Frequency $(\mathrm{Hz})$

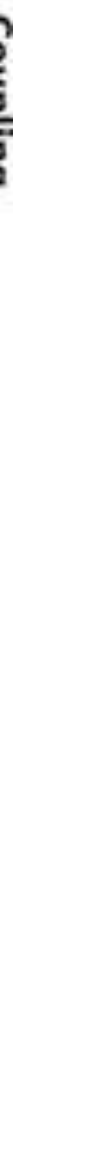


Click here to download 10. Supplementary Material: FigureS1.tif

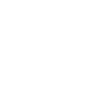
(1) $\sqrt{2}$ (1) (1) (1) (1) (1) (1) (1)

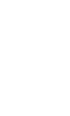

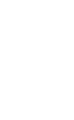
(n)

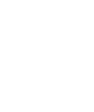
.

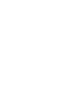

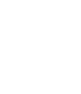

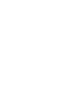

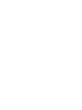

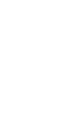
$\sqrt{n+2}+x^{2}$ $\sqrt{n+2}+x^{2}$ ( . (n) (n) $\sqrt{n+2}+x^{2}$ . . 列 . .

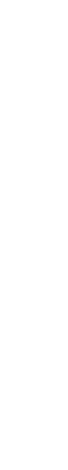

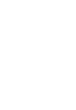
.

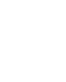

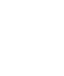
. 西 
Click here to download 10. Supplementary Material: FigureS2.tif

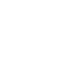

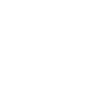

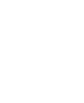

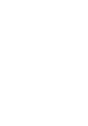

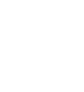
.

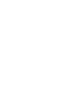
Material: Figures2.tif

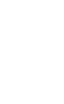
Material: FigureS2.tif

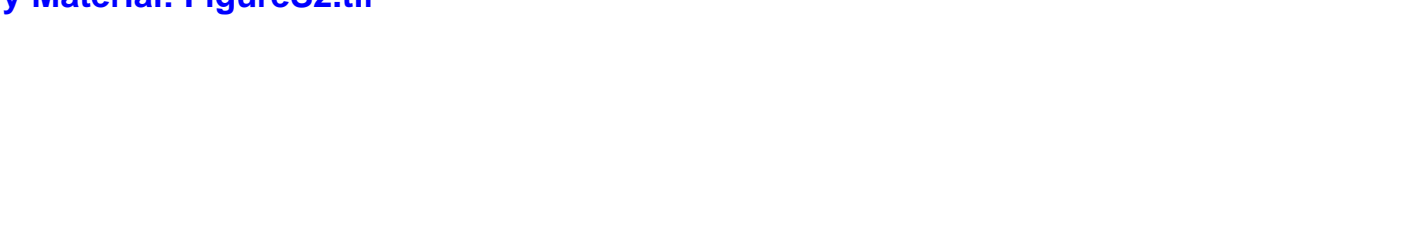

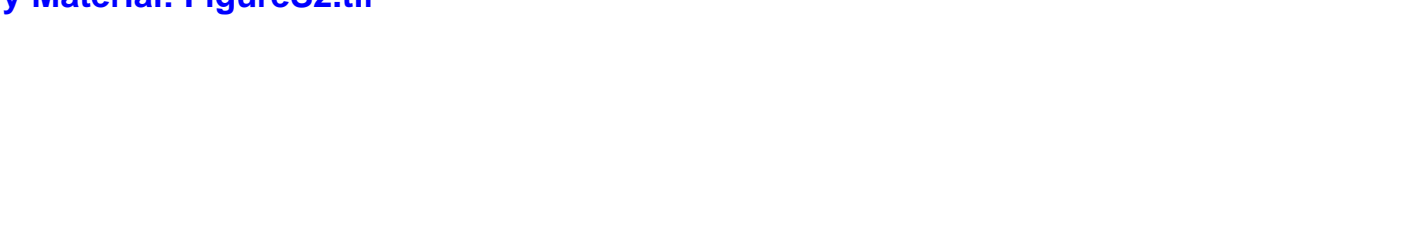
,

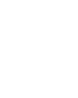
$-$ $-$ ,

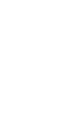

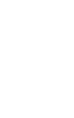
.

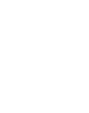

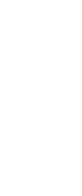

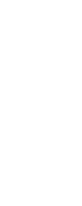
.

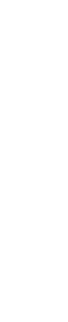

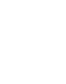

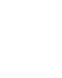

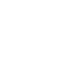

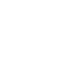

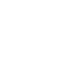
. 
Supplementary Figure S3
Click here to download 10. Supplementary Material: FigureS3.tif

if
更 .

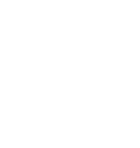
.

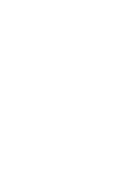
.

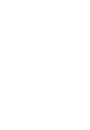
. (n) (n)

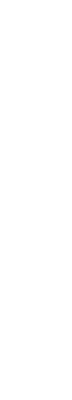

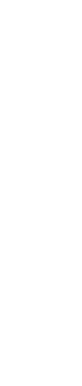

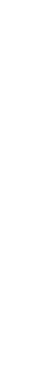
(1)

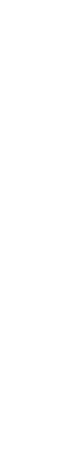
(1) (n) 西 (1)

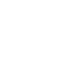


Supplementary Figure S4
Click here to download 10. Supplementary Material: FigureS4.tif

.

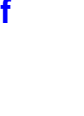

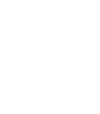

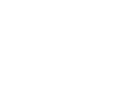

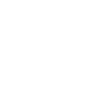

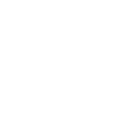

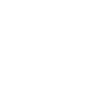

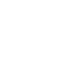


Supplementary Figure S5
Click here to download 10. Supplementary Material: FigureS5.tif

Supplementary Figure S5
Click here to download 10 . Supplementary Material: FigureS5.tif

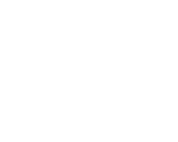

(1)
(1) (1)

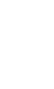
.

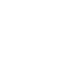

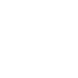
(1) (1) (1) (1) (1)

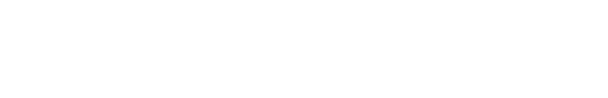
(1) (1) (1) (1) . (n)

(1)

(1)

(1) (1)

(1)

(1)

(1)

(1)

(1) (1) (1) (1) (1) (1) (1)

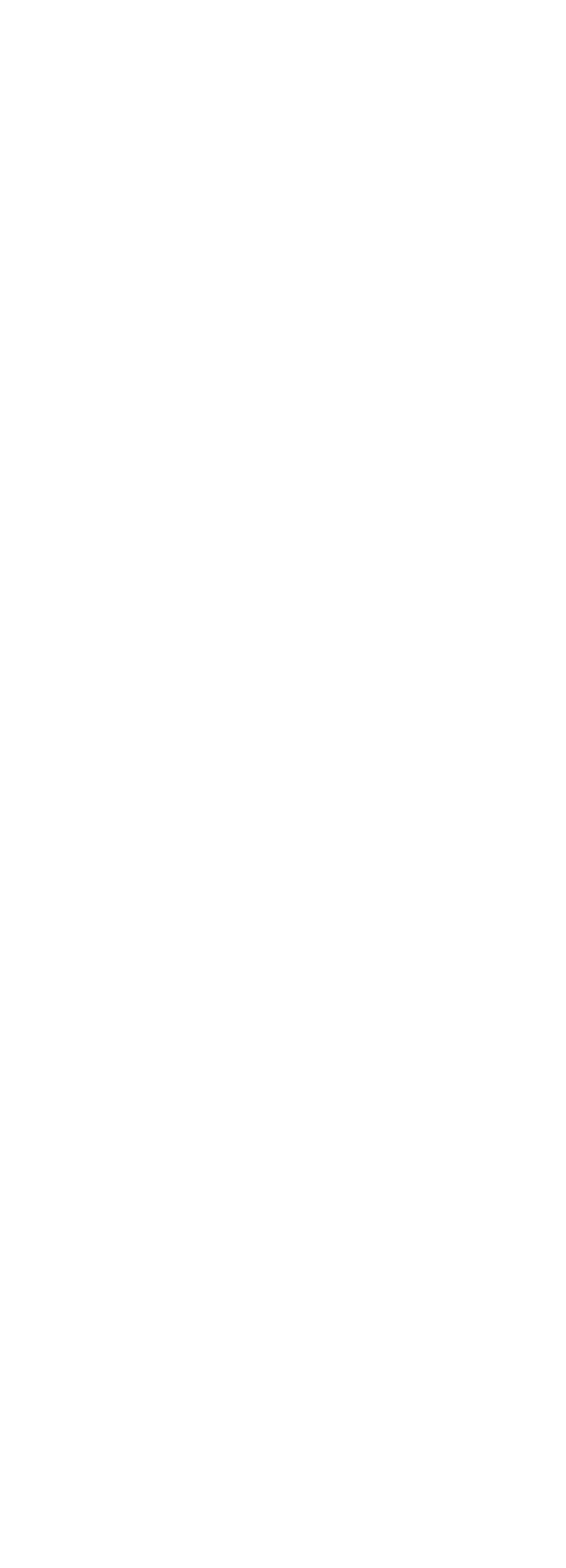

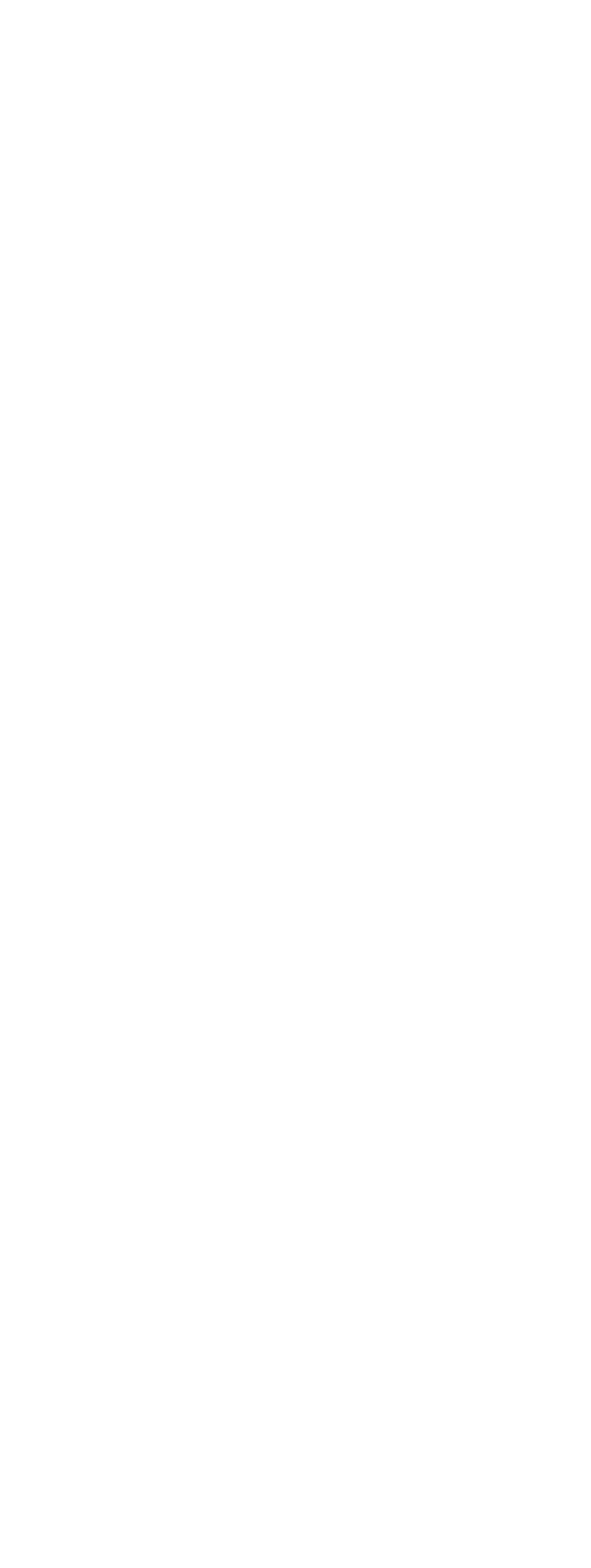

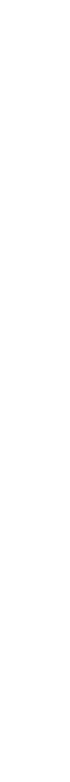
(1) 